\title{
$\left({ }^{6} \mathbf{L i}, d\right)$ and $\left({ }^{6} \mathbf{L i}, t\right)$ reactions on ${ }^{22} \mathrm{Ne}$ and implications for $s$-process nucleosynthesis
}

\author{
S. Ota,${ }^{1,2, *}$ G. Christian, ${ }^{1,3,4,5}$ W. N. Catford,${ }^{6}$ G. Lotay,${ }^{6}$ M. Pignatari,${ }^{7,8,9,2}$ U. Battino, ${ }^{10,2}$ \\ E. A. Bennett, ${ }^{1,4}$ S. Dede, ${ }^{1,4}$ D. T. Doherty, ${ }^{6}$ S. Hallam, ${ }^{6}$ F. Herwig, ${ }^{11,7,2}$ J. Hooker, ${ }^{1,4}$ C. \\ Hunt,${ }^{1,4}$ H. Jayatissa, ${ }^{1,4}$ A. Matta,${ }^{6}$ M. Moukaddam, ${ }^{6}$ E. Rao,${ }^{1,12}$ G. V. Rogachev,${ }^{1,4,5}$ \\ A. Saastamoinen, ${ }^{1}$ D. Scriven, ${ }^{1,4}$ J. A. Tostevin,${ }^{6}$ S. Upadhyayula,,${ }^{1,4}$ and R. Wilkinson ${ }^{6}$ \\ ${ }^{1}$ Cyclotron Institute, Texas A\&M University, College Station, TX 7r843, USA \\ ${ }^{2}$ NuGrid Collaboration, http: // nugridstars.org \\ ${ }^{3}$ Department of Astronomy $\&$ Physics, Saint Mary's University, Halifax, NS B3H 3C3, Canada \\ ${ }^{4}$ Department of Physics 8 Astronomy, Texas A\&M University, College Station, TX 77843, USA \\ ${ }^{5}$ Nuclear Solutions Institute, Texas AछM University, College Station, TX 7r843, USA \\ ${ }^{6}$ Department of Physics, University of Surrey, Guildford GU2 7XH, UK \\ ${ }^{7}$ Joint Institute for Nuclear Astrophysics - Center for the Evolution of the Elements, East Lansing, 48823, USA \\ ${ }^{8}$ E. A. Milne Centre for Astrophysics, Department of Physics and Mathematics, University of Hull, Hull HU6 7RX, UK \\ ${ }^{9}$ Konkoly Observatory, Research Centre for Astronomy and Earth Sciences, \\ Hungarian Academy of Sciences, Konkoly Thege M. $t$ 15-17, 1121, Budapest, Hungary \\ ${ }^{10}$ School of Physics and Astronomy, University of Edinburgh, EH9 3FD, UK \\ ${ }^{11}$ Department of Physics and Astronomy, University of Victoria, Victoria, BC V8P5C2, Canada \\ ${ }^{12}$ Department of Physics \& Astronomy, Rutgers University, New Brunswick, NJ, USA
}

(Dated: October 10, 2021)

\begin{abstract}
We studied $\alpha$ cluster states in ${ }^{26} \mathrm{Mg}$ via the ${ }^{22} \mathrm{Ne}\left({ }^{6} \mathrm{Li}, d \gamma\right){ }^{26} \mathrm{Mg}$ reaction in inverse kinematics at an energy of $7 \mathrm{MeV} /$ nucleon. States between $E_{x}=4-14 \mathrm{MeV}$ in ${ }^{26} \mathrm{Mg}$ were populated and relative $\alpha$ spectroscopic factors were determined. Some of these states correspond to resonances in the Gamow window of the ${ }^{22} \mathrm{Ne}(\alpha, \mathrm{n})^{25} \mathrm{Mg}$ reaction, which is one of the main neutron sources in the astrophysical $s$-process. Using our new ${ }^{22} \mathrm{Ne}(\alpha, \mathrm{n}){ }^{25} \mathrm{Mg}$ and ${ }^{22} \mathrm{Ne}(\alpha, \gamma){ }^{26} \mathrm{Mg}$ reaction rates, we performed new $s$-process calculations for massive stars and Asymptotic Giant Branch stars and compared the resulting abundances with the abundances obtained using other ${ }^{22} \mathrm{Ne}+\alpha$ rates from the literature. We observe an impact on the $s$-process abundances up to a factor of three for intermediate-mass AGB stars and up to a factor of ten for massive stars. Additionally, states in ${ }^{25} \mathrm{Mg}$ at $E_{x}<7.5 \mathrm{MeV}$ are identified via the ${ }^{22} \mathrm{Ne}\left({ }^{6} \mathrm{Li}, t\right){ }^{25} \mathrm{Mg}$ reaction for the first time. We present the $\left({ }^{6} \mathrm{Li}, t\right)$ spectroscopic factors of these states and note similarities to the $(d, p)$ reaction in terms of reaction selectivity.
\end{abstract}

\section{INTRODUCTION}

Most of the elements heavier than iron are made by neutron capture processes in stars, with additional minor contributions from other nuclear reactions such as photodisintegrations. In the solar system, about half of the abundances of heavy elements are made by the slow neutron-capture process ( $s$-process) [1], while most of the remaining abundances are made by the rapid neutroncapture process ( $r$-process) [2]. Additional contributions from the intermediate neutron-capture processes ( $i$-process) [3] are still a matter of debate [4], and explosive nucleosynthesis components from supernovae are expected to be potentially relevant only up to the Sr$\mathrm{Pd}$ region [5, 6]. Further additional contributions are expected to be made by the $p$-process, which feeds the neutron-deficient side of the isotopic chart beyond iron primarily by photodisntegration reactions and gives a significant contribution to the production of some isotopes of, e.g., Mo and $\mathrm{Ru}$ ( $p$-nuclides) in supernovae [7-9]. The $r$-process occurs in extreme stellar environments such as

\footnotetext{
*shuyaota@comp.tamu.edu
}

neutron star mergers $[10,11]$ and rare types of supernovae [2]. Its constituent reactions involve extremely neutron-rich nuclei far from stability $[12,13]$. In contrast, the $s$-process occurs during hydrostatic stellar evolution, and because of the lower neutron densities the $s$-process nucleosynthesis path proceeds along the valley of stability. As a result, the $s$-process can potentially be better constrained through accessing the relevant nuclear physics, e.g., Ref, [14], and stellar computational modeling [15]. By studying the isotopic pattern of the solar abundances for heavy $s$-process elements, three different $s$-process components have been identified. The main and the strong components are mostly formed in lowto-intermediate mass Asymptotic Giant Branch (AGB) stars in the He-rich intershell region [16] $\left(M / M_{\odot} \sim 1.5\right.$ 3 ), producing most of the solar $s$-process abundances in the $A \sim 90-209$ range - i.e., beyond the $N=50$ peak at ${ }^{88} \mathrm{Sr}[17,18]$. The 'weak' $s$-process components are made in massive stars $\left(M / M_{\odot} \gtrsim 8\right)$, during the convective He core and $\mathrm{C}$ shell burning phases. The neutron exposures optained in these conditions produce nuclides in the $A \sim 60-90$ mass region, possibly contributing also to the long-lived radioactive isotope ${ }^{60} \mathrm{Fe}[19-22]$.

The accurate knowledge of the rates of neutron- 
generating reactions and of relevant neutron-capture cross sections are crucial to move toward a complete understanding of $s$-process nucleosynthesis. Along with the ${ }^{13} \mathrm{C}(\alpha, n){ }^{16} \mathrm{O}$ reaction, ${ }^{22} \mathrm{Ne}(\alpha, n){ }^{25} \mathrm{Mg}$ has long been recognized as one of the most important $s$-process neutron sources [23, 24]. For the main s-process in AGB stars, most of the neutrons are made by the ${ }^{13} \mathrm{C}(\alpha, n){ }^{16} \mathrm{O}$ reaction. However, during the Thermal Pulse the ${ }^{22} \mathrm{Ne}(\alpha, n){ }^{25} \mathrm{Mg}$ reaction is partially activated for temperatures larger than about $0.25 \mathrm{GK}$, providing an additional neutron exposure and higher neutron densities [25]. In the He intershell region just below the $\mathrm{H}$ shell, ${ }^{12} \mathrm{C}$ is produced via triple- $\alpha$ reactions during the convective Thermal Pulses. The ${ }^{12} \mathrm{C}$ captures protons brought in the He-rich material by the Third-Dredge Up events, ultimately forming ${ }^{13} \mathrm{C}$ following decay of the generated ${ }^{13} \mathrm{~N}$, and creating the ${ }^{13} \mathrm{C}$-pocket, where the ${ }^{13} \mathrm{C}(\alpha, n){ }^{16} \mathrm{O}$ reaction activates the $s$-process in radiative conditions [16]. The following Thermal Pulse mixes the $s$-process rich material from the ashes of the ${ }^{13} \mathrm{C}$-pocket in the $\mathrm{He}$ intershell. The high neutron density generated by the ${ }^{22} \mathrm{Ne}(\alpha, n){ }^{25} \mathrm{Mg}$ reaction allows activation of several $s$ process branching points (e.g., ${ }^{95} \mathrm{Zr}$ ), modifying the isotopic pattern and producing isotopes not accessible during the neutron exposure in the ${ }^{13} \mathrm{C}$-pocket [26].

In the weak $s$-process, ${ }^{22} \mathrm{Ne}(\alpha, n){ }^{25} \mathrm{Mg}$ is the dominant neutron source throughout both the He core and the $\mathrm{C}$ shell over a range of stellar temperatures between about $0.25 \mathrm{GK}$ and $1 \mathrm{GK}[21,27-29]$. In C-shell burning, longlived ${ }^{60} \mathrm{Fe}$ is also made, thus contributing to the total ejected amount of this isotope [30]. The production of galactic ${ }^{60} \mathrm{Fe}$ is of significant interest since it is detected as a diffusive $\gamma$-ray source in the Galaxy [31] and its signature has been identified in the Early Solar System [32].

After many theoretical and experimental efforts over the past few decades, there still exist significant uncertainties in the stellar rate of ${ }^{22} \mathrm{Ne}(\alpha, n)^{25} \mathrm{Mg}$, as well as the competing reaction ${ }^{22} \mathrm{Ne}(\alpha, \gamma){ }^{26} \mathrm{Mg}$, e.g. as discussed in Ref [33]. The uncertainties are dominated by ambiguous strengths of some resonances in the Gamow window ( $T=0.2-0.3 \mathrm{GK}$, corresponding to $E_{x}=11.1-11.4 \mathrm{MeV}$ in $\left.{ }^{26} \mathrm{Mg}\right)$. This is due in large part to difficulties in isolating specific states due to the high level density of ${ }^{26} \mathrm{Mg}$ in this excitation region, as well as the low overall cross sections which challenge direct measurements. In the last decade, many new experiments targeting the role of ${ }^{26} \mathrm{Mg}$ resonances in ${ }^{22} \mathrm{Ne}(\alpha, n)^{25} \mathrm{Mg}$ have been published, building upon the knowledge of resonance properties accumulated in the 1980s and 1990s. These have indicated that the ${ }^{22} \mathrm{Ne}(\alpha, n){ }^{25} \mathrm{Mg}$ reaction is dominated by the resonance at $E_{x}=11.32 \mathrm{MeV}$, and possibly a second resonance in the $E_{x} \sim 11.15-11.17 \mathrm{MeV}$ region [34-36]. Additionally, Koehler [37] pointed out that a $2^{+}$resonance just above the neutron threshold, at $E_{x}=11.11 \mathrm{MeV}$ may dominate the rate at low temperatures. Massimi et al. later performed a precise measurement of the neutron and $\gamma$ ray partial widths of this state in a scan of $(n, \gamma)$ resonances using a Time-of-Flight neutron beam, confirming
Koehler's claim [38, 39].

In the $11.15-11.17 \mathrm{MeV}$ region, a candidate $1^{-}$state at $11.15 \mathrm{MeV}$ was originally considered as a potential contributor to both ${ }^{22} \mathrm{Ne}+\alpha$ capture reactions. Upper limits on $\Gamma_{\alpha}$ were set both from $\left({ }^{6} \mathrm{Li}, d\right)$ [40] and direct $(\alpha, n)[36]$ measurements. However, this state was later shown to be $1^{+}$(and hence non-natural parity) by $\left(\gamma, \gamma^{\prime}\right)$ experiments by Longland et al. [33, 41]. Talwar et al. later suggested the presence of a natural parity $\left(1^{-}\right.$or $2^{+}$) resonance at $11.17 \mathrm{MeV}$, with a large $\gamma$-ray partial width, indicating an enhanced $(\alpha, \gamma)$ cross section that suppresses the total neutron production by consuming ${ }^{22} \mathrm{Ne}$ in competition with the weaker $(\alpha, n)$ channel [42]. Adsley et al. performed high energy-resolution measurements of ${ }^{26} \mathrm{Mg}\left(\alpha, \alpha^{\prime}\right),{ }^{26} \mathrm{Mg}\left(p, p^{\prime}\right)$, and ${ }^{26} \mathrm{Mg}\left(d, d^{\prime}\right)$, and provided important properties of these resonances above such as excitation energy with high precision [43, 44]. Lotay et al. observed a strong $\gamma$ transition from the 11.17 $\mathrm{MeV}$ state [45]. This result was consistent with Talwar et al.'s claim of a large $\Gamma_{\gamma}$, but suggestive of a higher spin state $(J=2-6)$. Most recently, Jayatissa et al. failed to observe a state at $11.17 \mathrm{MeV}$ in a measurement of the ${ }^{22} \mathrm{Ne}\left({ }^{6} \mathrm{Li}, d\right){ }^{26} \mathrm{Mg}$ reaction at sub-Coulomb energies [46]. This reaction mechanism is likely to be capable of populating natural-parity states only with $J \leq 2$, suggesting that the state observed at $11.17 \mathrm{MeV}$ by Talwar et al. is high spin $(J \geq 3)$. All together, the latest experimental results indicate that the $11.17 \mathrm{MeV}$ state has a large $\Gamma_{\gamma}$ and high spin $(J \geq 3)$, thus making its contribution to the $s$-process negligible.

For the remaining $11.32 \mathrm{MeV}$ resonance, the $(\alpha, \gamma)$ resonance strength is well established, with earlier measurements [34, 47] recently confirmed by the new study of Hunt et al. [48], which reported $\omega \gamma=46 \pm 11 \mu \mathrm{eV}$. The weighted average of published direct-measurement $(\alpha, \gamma)$ strengths for this resonance is $37 \pm 4 \mu \mathrm{eV}$. The $(\alpha, n)$ strength of this resonance is more uncertain, with the results of direct measurements in poor statistical agreement [33]. A recent letter published by the present authors determined the $\Gamma_{n} / \Gamma_{\gamma}$ branching ratio of this resonance via the ${ }^{22} \mathrm{Ne}\left({ }^{6} \mathrm{Li}, d\right){ }^{26} \mathrm{Mg}$ reaction in inverse kinematics [49]. This was done by observing the decay of the recoil ${ }^{26} \mathrm{Mg}$ into either ${ }^{26} \mathrm{Mg}+\gamma$ or ${ }^{25} \mathrm{Mg}+n$. Normalizing to the $(\alpha, \gamma)$ strength of $37 \pm 4 \mu \mathrm{eV}$, this established an $(\alpha, n)$ strength of $42 \pm 11 \mu \mathrm{eV}$, which is a factor $\sim 3$ smaller than the past direct measurements. Based on the results of recent experiments, the $11.32 \mathrm{MeV}$ resonance appears to be the main contributor to the stellar rate across the important temperature range for the $s$-process. At the same time, the $11.17 \mathrm{MeV}$ resonance observed by Talwar et al. is unlikely to contribute to the stellar reaction. The main outstanding uncertainty concerns the $11.11 \mathrm{MeV}$ resonance identified by Massimi et al. [39]. This state has the potential to dominate the $(\alpha, n)$ rate at low temperatures (below $\sim 0.2 \mathrm{GK}$ ). The Monte Carlo rate calculations presented in Ref. [49], which sample the possible strengths from a Porter-Thomas distribution, indicate a large uncertainty in the contribution of this resonance to 
the stellar rate in this temperature range.

In the present paper, we significantly expand our first letter paper [49] wherein we reported $\Gamma_{n} / \Gamma_{\gamma}=1.14 \pm 0.26$ at $E_{x}=11.32 \mathrm{MeV}$. In particular, we present a complete description of the experimental setup, which combined the TIARA Si detector array with four HPGe detectors and the MDM spectrometer at Texas A\&M University. Additionally, we present an analysis of the complete range of strong $\alpha$ cluster states in ${ }^{26} \mathrm{Mg}$ populated in the ${ }^{22} \mathrm{Ne}\left({ }^{6} \mathrm{Li}, d\right){ }^{26} \mathrm{Mg}$ reaction, as well as states in ${ }^{25} \mathrm{Mg}$ observed in the ${ }^{22} \mathrm{Ne}\left({ }^{6} \mathrm{Li}, t\right){ }^{25} \mathrm{Mg}$ reaction. Finally, we present the results of a large scale computer simulation, which determines the effect on $s$-process abundances of replacing the literature values of the ${ }^{22} \mathrm{Ne}(\alpha, n)^{25} \mathrm{Mg}$ and ${ }^{22} \mathrm{Ne}(\alpha, \gamma){ }^{26} \mathrm{Mg}$ rates with the new rates presented in Ref. [49]. For the simulations, we used a multi-zone postprocessing code, MPPNP [50], to study the sensitivity at various stellar masses and initial metallicities. The simulations also demonstrate, in a more general way, the sensitivity of the abundance to the ambiguous reaction rates, by varying the $\alpha$ cluster strength of relevant ${ }^{22} \mathrm{Ne}+\alpha$ resonances.

\section{EXPERIMENTAL SETUP}

We performed the experiment at the Texas A\&M University Cycloton Institute, using the K150 cyclotron to produce a beam of $154 \mathrm{MeV}{ }^{22} \mathrm{Ne}^{(7+)}$ ions. We studied the $\left({ }^{6} \mathrm{Li}, d\right)$ reaction using ${ }^{22} \mathrm{Ne}$ beam in inverse kinematics, impinging the beam onto an isotopically-enriched ${ }^{6} \mathrm{LiF}$ target mounted on a carbon backing. We detected both the deuteron ejectiles and unambiguously identified ${ }^{25,26} \mathrm{Mg}$ recoils in coincidence, along with the $\gamma$ rays resulting from the de-excitation of states populated in ${ }^{25,26} \mathrm{Mg}$. Deuterons, ${ }^{25,26} \mathrm{Mg}$ recoils, and $\gamma$-decays were measured with the TIARA Si detector array, the MDM spectrometer, or an array of four closely-packed HPGe detectors, respectively. A detailed description of each of these components is given in the following sub-sections.

\section{A. TIARA}

TIARA consists of two sets of Si detectors called the "Hyball" and "Barrel", respectively [51]. The Hyball consists of six wedge-shaped, double-sided Si detectors with 16 rings and 8 sectors for each. The effective area of each detector covers an azimuthal angle of $\phi=54.8^{\circ}$. Together, the six wedges form an annular shape with inner and outer radii of $32.6 \mathrm{~mm}$ and $135.0 \mathrm{~mm}$, respectively. The Hyball was placed at $15 \mathrm{~cm}$ upstream from the target position and thus detected ejectiles emitted at laboratory polar angles from $\theta_{l a b}=145-168^{\circ}$ (note that angles from $\theta_{\text {lab }}=138$ to $\sim 145^{\circ}$ were not illuminated in the present experiment because they were shadowed by the Barrel detector). The Hyball consists of a single active layer of Si with a nominal thickness of $400 \mu \mathrm{m}$.
The Barrel consists of eight resistive charge division $\mathrm{Si}$ detectors forming an octagonal barrel around the beam axis, surrounding the target and covering polar angles from $\theta_{l a b}=40-145^{\circ}$. Each Barrel detector is segmented by four strips in the azimuthal $(\phi)$ direction. The zposition (along the beam axis) of incident particles is determined from the ratio of deposited energies in the upstream and downstream electrodes, after correcting for the ballistic deficit. The z-axis position along the barrel directly corresponds to $\theta_{l a b}$ in the present geometry. The expected z-position resolution is $1 \mathrm{~mm}$ (FWHM) [52], leading to the $\theta_{l a b}$ resolution better than $2^{\circ}$. Further details about energy and angle measurements in the Barrel can be found in, e.g. Ref. [52]. The resistive-strip layer of the Barrel ("Inner Barrel") has a nominal thickness of $400 \mu \mathrm{m}$ and is backed by an unsegmented outer layer ("Outer Barrel") with $1 \mathrm{~mm}$ active thickness. Thus, particles which punch through the Inner Barrel can be identified by the conventional $\Delta E-E$ method (see Figure $2 \mathrm{~b}$ ).

From measurements using multi-nuclide $\left({ }^{239} \mathrm{Pu},{ }^{241} \mathrm{Am}\right.$ and $\left.{ }^{244} \mathrm{Cm}\right) \alpha$ sources, the intrinsic energy resolutions of the Hyball and Barrel were determined to be 40 and $100 \mathrm{keV}$ FWHM, respectively. These correspond to 180$280 \mathrm{keV}$ and $400-600 \mathrm{keV}$ resolutions in the center-ofmass system for the ${ }^{22} \mathrm{Ne}\left({ }^{6} \mathrm{Li}, d\right)$ and ${ }^{22} \mathrm{Ne}(d, p)$ reactions. Center-of-mass resolutions in the barrel are dominated by the poor angular resolution $\delta_{\theta_{C M}} \sim 1^{\circ}$, relative to the steep slope of the $E_{l a b}$ vs. $\theta_{l a b}$ kinematic curves in the corresponding angular region. As the Barrel's energy resolution is insufficient to separate states in the ${ }^{26} \mathrm{Mg}$ excitation energy spectrum, and furthermore because the ${ }^{26} \mathrm{Mg}$ in coincidence with most of deuterons detected by the Barrel are beyond the MDM acceptance, the barrel was used mostly for monitoring elastic scattering.

\section{B. MDM spectrometer}

The MDM spectrometer downstream of the target transports particles scattered at forward angles less than $\theta_{l a b}= \pm 2^{\circ}$ in both the dispersive (x) and non-dispersive (y) planes. Transported particles are detected in the Oxford detector [53, 54]. The Oxford detector consists of three ionization detector zones and four wire proportional counter zones. Energy deposit signals in the two ionization zones in the downstream side are amplified with Micromegas plates [55]. The isobutane gas pressure was adjusted so that the incident recoil ions of interest are stopped in the last ionization zone (35 torr for $\left({ }^{6} \mathrm{Li}, d\right)$ and 70 torr for $(d, p)$ reactions, respectively). Thus recoil particles were identified by $\Delta \mathrm{E}$-E method with high energy-resolution. The resistive wires provided the particle positions and trajectories with a few $\mathrm{mm}$ resolution in X-z plane. Thus particles incident on the Oxford detector are identified in mass $(\mathrm{A})$ and atomic number $(\mathrm{Z})$ from the $\mathrm{E}, \Delta \mathrm{E}$, and $\mathrm{x}$-position information at the focal plane position, which is approximately located at the second resistive wire. The charge state (Q) distribution 


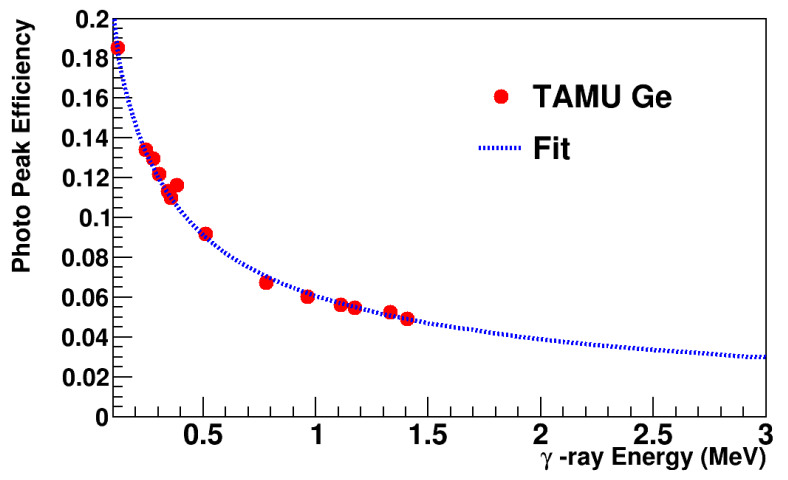

FIG. 1. Total photopeak efficiency (sum of efficiency by each clover), together with data obtained using some conventional $\gamma$ sources. Note the error bars are smaller than the symbol size.

of ${ }^{26} \mathrm{Mg}$ after passing the target is estimated to be dominated by $12^{+}[56]$. Thus the magnet rigidity was set to accept ${ }^{25,26} \mathrm{Mg}$ recoils with charge $Q=12$.

\section{HPGe array}

Four closely-packed HPGe clovers [57] were placed at a distance of about $10 \mathrm{~cm}$ from the target position (distance is quoted to the detector surface). Each clover consists of four crystals and is also electrically segmented into three sections toward the beam direction (downstream, middle, upstream). The segmentation information was used to correct for Doppler shift of the $\gamma$ rays emitted from beam-like recoils $(\beta \sim 0.1)$. The energy resolution achieved for beam recoil $\gamma$-rays is $3 \%$ at FWHM for $1 \mathrm{MeV} \gamma$ rays. This is primarily limited by angular resolution in the Doppler correction. Photopeak efficiency was measured using some conventional $\gamma$-ray sources. Absolute efficiency was determined from the coincidence measurements of ${ }^{60} \mathrm{Co} \gamma$-rays $(1173$ and $1332 \mathrm{keV}$ ), and ${ }^{22} \mathrm{Na}$ pair-production $\gamma$-rays $(511$ $\mathrm{keV})$. The total efficiency curve was then determined with ${ }^{152} \mathrm{Eu}$ and ${ }^{133} \mathrm{Ba}$ source measurements, normalizing to the absolute efficiency measurements. Figure 1 shows the measured photopeak efficiency for this setup, together with a fit to the following function $\left(E_{\gamma}>0.1\right.$ $\mathrm{MeV}): \Sigma_{i=1}^{4} \exp \left(a_{i}+b_{i} \times \ln \left(E_{\gamma} / E_{0}\right)+c_{i} \times \ln \left(E_{\gamma} / E_{0}\right)^{2}\right)$ [58], where a fixed reference energy $E_{0}$ is $1 \mathrm{MeV}$ and the average values of $a_{i}, b_{i}, c_{i}$ for each clover $i$ are -4.1979 , -0.6096 , and -0.0353 , respectively.

The distance from the chamber surface is about $1 \mathrm{~cm}$. Because of the proximity to the target position, photopeak $\gamma$-ray detection efficiencies achieved in this geometry are $23.5 \%, 9 \%$, and $6 \%$, at 100,500 , and $1 \mathrm{MeV}$, respectively. The photopeak efficiency was also estimated with the Geant4 simulations, and we confirmed that the fitted and simulated efficiencies agree with each other up to $4 \mathrm{MeV}$ within the $20 \%$ uncertainty. The measured ef- ficiency curve is valid up to $\sim 4 \mathrm{MeV}$; for higher energies, pair production begins to dominate and the extrapolated efficiency curve is not reliable. While we did attempt to measure $\gamma$ rays up to $\sim 11 \mathrm{MeV}$, using a dual-gain amplifier system, no clear signals in the higher-energy regime were observed due to the limited statistics. Hence we limited our analysis to $\gamma$ rays below $\sim 3 \mathrm{MeV}$, where the measured efficiency curve is reliable.

\section{Test measurement with ${ }^{22} \mathrm{Ne}(d, p)$}

To test the reliability of our experimental setup, we performed a measurement of ${ }^{22} \mathrm{Ne}(d, p)$ reaction, whose cross sections, $J^{\pi}$, and spectroscopic factors are well determined by Refs. [59, 60]. The beam impinged on a $500 \mu \mathrm{g} / \mathrm{cm}^{2} \mathrm{CD}_{2}$ target with the intensity of $1 \mathrm{pnA}$. The outgoing target-like protons were detected in the Hyball which measured their kinetic energy and angle with respect to the incoming beam. The reconstructed deuteron momenta were used to determine the excitation energy of ${ }^{23} \mathrm{Ne}$ states using the missing mass technique. Elastically scattered target nuclei were detected in the TIARA Si barrel array and used to continuously monitor the incoming beam rate.

Figure 2 shows an angle versus energy plot, in which theoretically calculated kinematic curves are also drawn. For $(d, d)$ elastic scattering events, we can see the expected correlation between the deuteron energy and scattering angles. The location of the elastic scattering kinematic curves was used to determine the position of the incoming beam, by comparing theoretical curves over a range of incident beam positions using a $\chi^{2}$ minimization technique. From this procedure, we obtained a beam position of $\mathrm{x}, \mathrm{y}, \mathrm{z}=-2,-1,4 \mathrm{~mm}$, which was used in later analysis of $(d, p)$ and $\left({ }^{6} \mathrm{Li}, d\right)$ data as well. The beam size was also evaluated from the observed elastic scattering kinematic curves, and determined to have a FWHM of $4-5 \mathrm{~mm}$. This agrees with the size determined from the luminescence produced by impinging the beam onto a phosphor-coated viewer plate.

The elastic scattering cross sections observed in the Barrel detector were compared with optical model calculations to determine the absolute beam + target luminosity normalization. A variety of available optical potential models [59-62] were used to calculate the elastic cross sections using the FRESCO code [63] and compared with our experimental data. The optical potential by Daehnick et al. [61] best reproduces the shape of our elastic cross section data and the beam intensity was normalized using this potential.

The ${ }^{22} \mathrm{Ne}(d, p)$ excitation spectrum and cross sections for some low-lying states are shown in Figure 3, together with DWBA calculations from the TWOFNR code [64] using the optical potentials from Refs. [61] and [65] for the deuteron and proton channels, respectively, assuming known $J^{\pi}$. Our data are well reproduced by the model calculations. Extracted spectroscopic factors are listed in 
TABLE I. Obtained spectroscopic factors for some low-lying states of ${ }^{23} \mathrm{Ne}$ observed in ${ }^{22} \mathrm{Ne}(d, p)$ reaction, compared with values from past measurements.

\begin{tabular}{ccccc}
\hline \hline$E_{x}(\mathrm{keV})$ & $J^{\pi}$ & $S$ (present) & $S$ (Ref. [60]) & $S$ (Ref. [59]) \\
\hline$G S$ & $5 / 2^{+}$ & $0.25(5)$ & 0.22 & 0.24 \\
1016 & $1 / 2^{+}$ & $0.58(12)$ & 0.70 & 0.40 \\
2315 & $5 / 2^{+}$ & $0.022(5)$ & $0.05(1)$ & 0.07 \\
\hline \hline
\end{tabular}

Table I. These values agree with past measurements, thus confirming that our measurement system works well.

The ${ }^{22} \mathrm{Ne}(d, p)$ data were also used to determine the intrinsic efficiency of the Oxford detector for detecting beam-like recoils. This was measured to be $80.0 \pm 2.0 \%$ by dividing the number of proton $+{ }^{23} \mathrm{Ne}$ coincidence events by the number of proton singles events. For these calculations, the MDM angular acceptance was taken to be $100 \%$ since recoils in coincidence with the Hyball protons are emitted within the $\pm 2^{\circ}$ entrance aperture. The MDM angular acceptance declines with decreasing proton scattering angle, dropping below $100 \%$ for events detected in the Barrel. For these events, the coincidence efficiency was estimated using Geant4 with the NPTOOL interface [66]. This efficiency correction for Barrel events is included in the cross sections shown in Figure 3.

The ${ }^{22} \mathrm{Ne}(d, p)$ data were also used to test and optimize our particle- $\gamma$ coincidence measurements. Because of Doppler broadening, the $\gamma$-ray energy resolution is strongly affected by the accuracy of the HPGe detector positions. Hence the position of each HPGe detector was systematically varied, and the position resulting in the narrowest Doppler-corrected energy resolution was taken for the final analysis. The photopeak efficiencies for selected $\gamma$-ray transitions observed in coincidence with $(d, p)$ events were also used to confirm the accuracy of our source-determined efficiency curve out to high $\gamma$-ray energies $(\sim 4 \mathrm{MeV})$.

\section{III. ${ }^{22} \mathrm{NE}+{ }^{6} \mathrm{LI}$ EXPERIMENT}

For the ${ }^{22} \mathrm{Ne}+{ }^{6} \mathrm{Li}$ portion of the experiment, the ${ }^{22} \mathrm{Ne}$ beam impinged on a $30 \mu \mathrm{g} / \mathrm{cm}^{2}{ }^{6} \mathrm{LiF}$ target $\left(95.0 \%{ }^{6} \mathrm{Li}\right.$ purity) with a $10 \mu \mathrm{g} / \mathrm{cm}^{2}$ carbon backing, with an intensity of $3 \mathrm{pnA}$. Recoil particle identification plots based on $E-\Delta E$ measurements in the Oxford detector, as well a plot of the ${ }^{26} \mathrm{Mg}$ excitation energy spectra gated on ${ }^{25} \mathrm{Mg}$ and ${ }^{26} \mathrm{Mg}$ recoils can be found in Ref. [49]. Unlike the $(d, p)$ measurements, the kinematic locus of ${ }^{22} \mathrm{Ne}+{ }^{6} \mathrm{Li}$ elastic scattering events is contaminated by background from elastic scattering on the fluorine and carbon contained in the target. Therefore, we focused on determining relative $\alpha$-particle strengths, normalized to the well-constrained state at $E_{x}=11.32 \mathrm{MeV}$ (see Ref. [49]). Note the Barrel was not used for the ${ }^{22} \mathrm{Ne}+{ }^{6} \mathrm{Li}$ reaction analysis due to its poor excitation energy resolution.
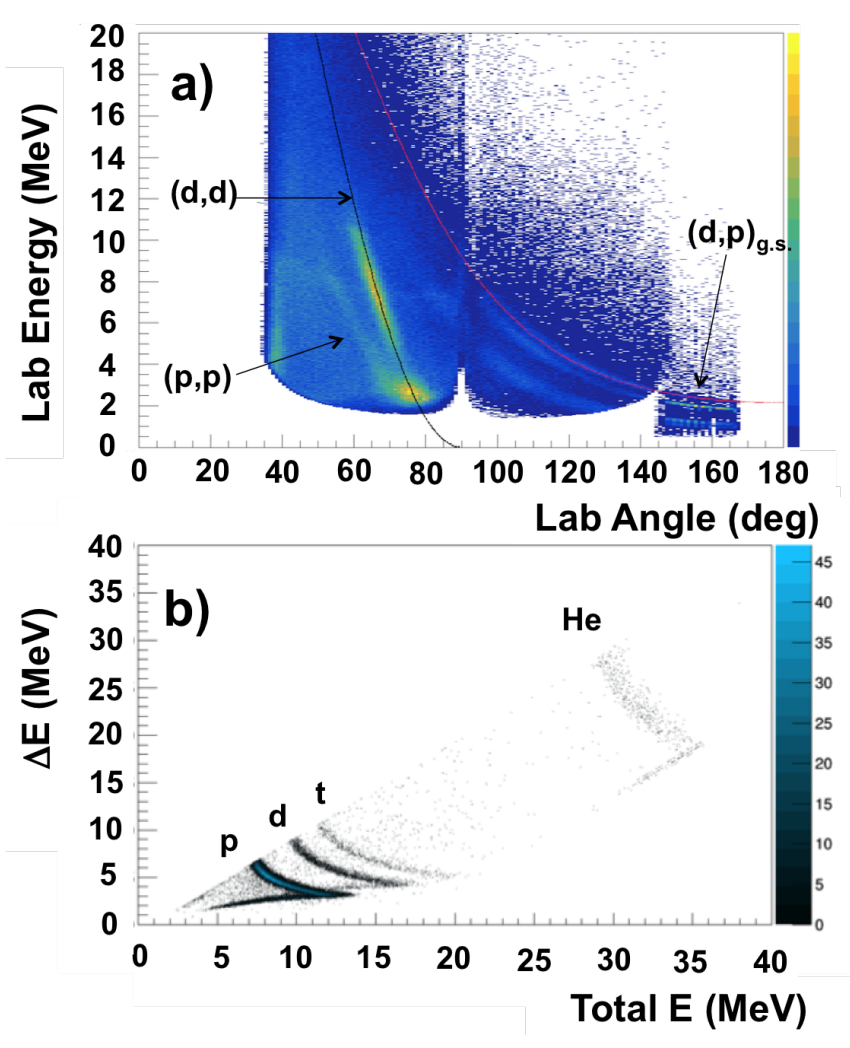

FIG. 2. a) Energy versus scattering angle plot from ${ }^{22} \mathrm{Ne}+\mathrm{CD}_{2}$. Theoretical elastic $(d, d)$ and $(d, p)$ ground state kinematic lines are shown together. The elastic $(p, p)$ line is because of contaminants in the target. b) $E-\Delta E$ plot from a Barrel detector, where protons, deuterons, tritons, and $\mathrm{He}$ are observed.

\section{A. Recoil energy and focal plane position}

Figure 4 shows the correlation of the recoil particle positions at the focal plane and ${ }^{26} \mathrm{Mg}$ excitation energies calculated from the Hyball signals assuming the ${ }^{22} \mathrm{Ne}\left({ }^{6} \mathrm{Li}, d\right){ }^{26} \mathrm{Mg}$ reaction. Clear correlations are seen in both figures, which are gated on ${ }^{25,26} \mathrm{Mg}$ recoils, respectively. These correlated loci correspond to two separate binary reaction mechanisms as explained below. Since the Hyball detector does not have particle identification capability, this correlation is essential to separate the two binary reactions observed in this study. The observed correlations occur because the ${ }^{25,26} \mathrm{Mg}$ recoils have lower kinematic energies when they are more highly excited in the binary reactions. Thus recoils with increasing excitation energy move towards the lower-rigidity (decreased $x$ position) side of the focal plane. It is also noticeable that the correlation disappears from ${ }^{26} \mathrm{Mg}$ events when the excitation energy becomes greater than the neutron separation energy $(11.09 \mathrm{MeV})$. Instead, the excited ${ }^{26} \mathrm{Mg}$ decays into ${ }^{25} \mathrm{Mg}+n$. This process gives a substantial momentum kick to ${ }^{25} \mathrm{Mg}$ following the neutron decay, which effectively destroys the kinematic correlations especially for large neutron decay energies (e.g., $E_{x}>12$ 

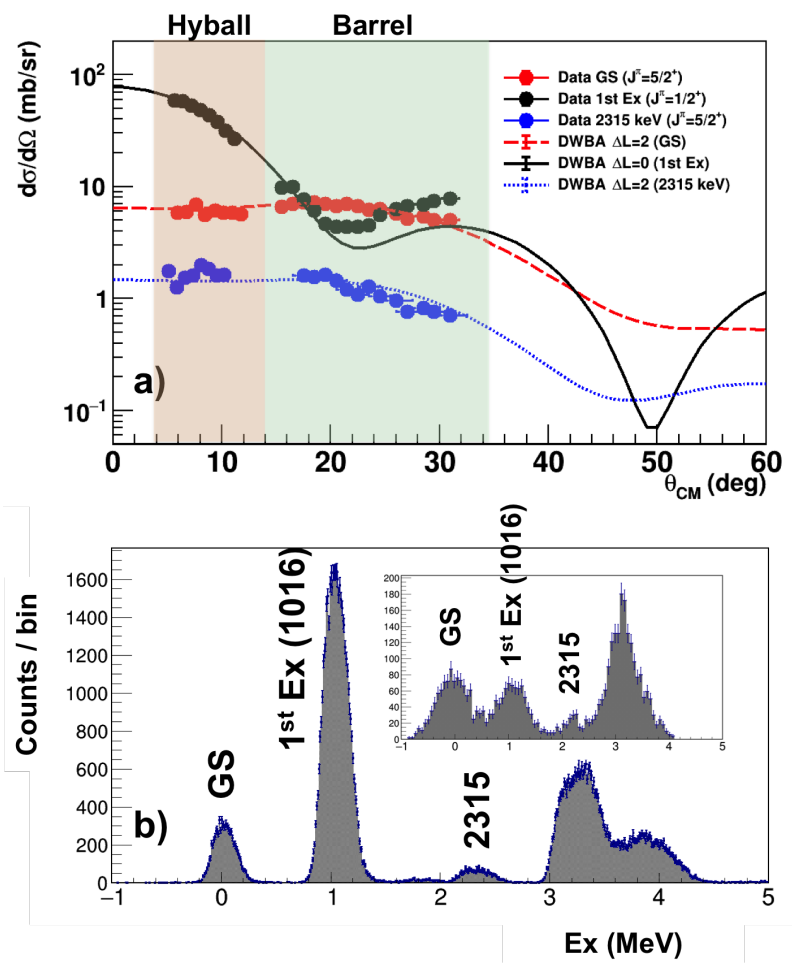

FIG. 3. a) Angular differential cross sections of ${ }^{22} \mathrm{Ne}(d, p)$ reactions for populating low-lying states of ${ }^{23} \mathrm{Ne}$. b) Excitation spectra of ${ }^{23} \mathrm{Ne}$ from the Hyball at $\theta_{C M}=5-12^{\circ}$ (whole detector). Inset: Barrel at $\theta_{C M}=18-19^{\circ}$.

$\mathrm{MeV})$.

If only ${ }^{22} \mathrm{Ne}\left({ }^{6} \mathrm{Li}, d\right)^{26} \mathrm{Mg}$ reactions were present, it would be expected that the $x-E_{x}$ plot gated on ${ }^{25} \mathrm{Mg}$ events would display less distinctive kinematic correlation, as all events would arise from the ${ }^{22} \mathrm{Ne}\left({ }^{6} \mathrm{Li}, d\right){ }^{26} \mathrm{Mg} \rightarrow{ }^{25} \mathrm{Mg}+n$ process described above. However, it is clear from Figure 4(b) that a correlated locus occurs in the high excitation energy and the large positive $x$-position side of the plot. This correlation occurs because these events arise from the ${ }^{22} \mathrm{Ne}\left({ }^{6} \mathrm{Li}, t\right)^{25} \mathrm{Mg}$ reaction. The association of these events with the $\left({ }^{6} \mathrm{Li}, t\right)$ reaction channel is confirmed by calculating the excitation energy assuming the $\left({ }^{6} \mathrm{Li}, t\right)$ reaction mechanism. When calculated as such, the excitation energy spectrum starts from $0 \mathrm{MeV}$ as would be expected (see Figure 5). This reaction mechanism is further discussed in Section III B.

In the figures, background events can be seen behind the correlated loci of binary reactions. These events are mostly due to protons arising from compound nuclear reactions. We confirmed this by constructing the same plots using Barrel-MDM coincidence events. In the barrel, light-particle identification is possible using the $\Delta E$-E method (see 2(b)), and the observed uncorrelated events were thus identified to be protons. In the binaryreaction analysis, the shape of the proton background was estimated from the uncorrelated events located near the binary events in Figure 4. These background contri- butions composed $\sim 5-10 \%$ of the total events, and were subtracted in bulk in the final excitation energy spectrum.

\section{B. ${ }^{22} \mathrm{Ne}\left({ }^{6} \mathbf{L i}, t\right){ }^{25} \mathrm{Mg}$ reaction}

We analyzed the ${ }^{22} \mathrm{Ne}\left({ }^{6} \mathrm{Li}, t\right){ }^{25} \mathrm{Mg}$ data to identify the states in ${ }^{25} \mathrm{Mg}$ that are strongly populated in this reaction and determine their spectroscopic factors. To date, there are no published data on the ${ }^{22} \mathrm{Ne}\left({ }^{6} \mathrm{Li}, t\right){ }^{25} \mathrm{Mg}$ reaction and hence this represents the first analysis of states in ${ }^{25} \mathrm{Mg}$ populated through this reaction mechanism. However, the $\left({ }^{6} \mathrm{Li}, t\right)$ reaction mechanism has been studied on a variety of light-to-medium mass targets including ${ }^{13} \mathrm{C} \quad[68-70],{ }^{16} \mathrm{O} \quad[71-73],{ }^{24} \mathrm{Mg},{ }^{28} \mathrm{Si},{ }^{40} \mathrm{Ca}$, ${ }^{54,56} \mathrm{Fe}$, and ${ }^{58} \mathrm{Ni}[74,75]$, as a potential method for spectroscopy. Most of these studies were carried out in the 1970s or earlier except for the recent ${ }^{13} \mathrm{C}\left({ }^{6} \mathrm{Li}, t\right)$ study of Ref. [70]. Despite the large binding energy (15.8 MeV), appreciable $t+{ }^{3} \mathrm{He}$ clustering in the ground state of ${ }^{6} \mathrm{Li}$ is reported in those past studies.

A few potential direct reaction mechanisms were proposed in the earlier $\left({ }^{6} \mathrm{Li}, t\right)$ studies. One is that the proton pair goes into the lowest accessible orbits, while the odd neutron is transferred into single-particle states (in the present experiment, this would populate a similar set of ${ }^{25} \mathrm{Mg}$ states as $\left.{ }^{24} \mathrm{Mg}(d, p)^{25} \mathrm{Mg}\right)$ [75]. Another possibility is that one proton is transferred to the lowestavailable orbital and the remaining proton-neutron pair is transferred as in the $(\alpha, d)$ reaction (analogous to ${ }^{23} \mathrm{Na}(\alpha, d){ }^{25} \mathrm{Mg}$ in the present case) [69]. Finally, an analogous mechanism to direct $(\alpha, n)$ reactions with transfer of a ${ }^{3} \mathrm{He}$ cluster $\left({ }^{22} \mathrm{Ne}(\alpha, n){ }^{25} \mathrm{Mg}\right.$ at present) has also been proposed [75]. However, the reaction's selectivity in populating the specific levels observed in the past experiments $[68,69,71,74,75]$ are not universally explained by any of the above mechanisms.

The background-subtracted ${ }^{25} \mathrm{Mg}$ excitation spectrum from the present experiment is shown in Figure 5. A number of narrow small peaks and some large peaks are evident, indicating the strongly selective population of discrete states through a direct transfer mechanism. This is consistent with existing $\left({ }^{6} \mathrm{Li}, t\right)$ studies. Due to the low statistics, we were unable to perform a particle- $\gamma$ coincidence analysis to unambiguously identify the populated states through their $\gamma$-ray transitions. Instead, we made a tentative assignment of the populated states based purely on excitation energies observed from $\left({ }^{6} \mathrm{Li}, t\right)$ (the excitation energy uncertainty is $\pm 20 \mathrm{keV}$ ). Due to the energy resolution of the present setup $(\sim 200 \mathrm{keV})$, it is possible that the observed peaks may contain transitions to two or more states. Hence, for each peak we have performed a separate analysis of all candidate states that overlap in energy.

The strongest peaks in the observed spectrum appear at near $E_{x}=3413\left(J^{\pi}=3 / 2^{-}\right), 3970\left(7 / 2^{-}\right), 4277\left(1 / 2^{-}\right)$, and $7286\left(7 / 2^{-}\right) \mathrm{keV}$, respectively. Notably, these peaks 


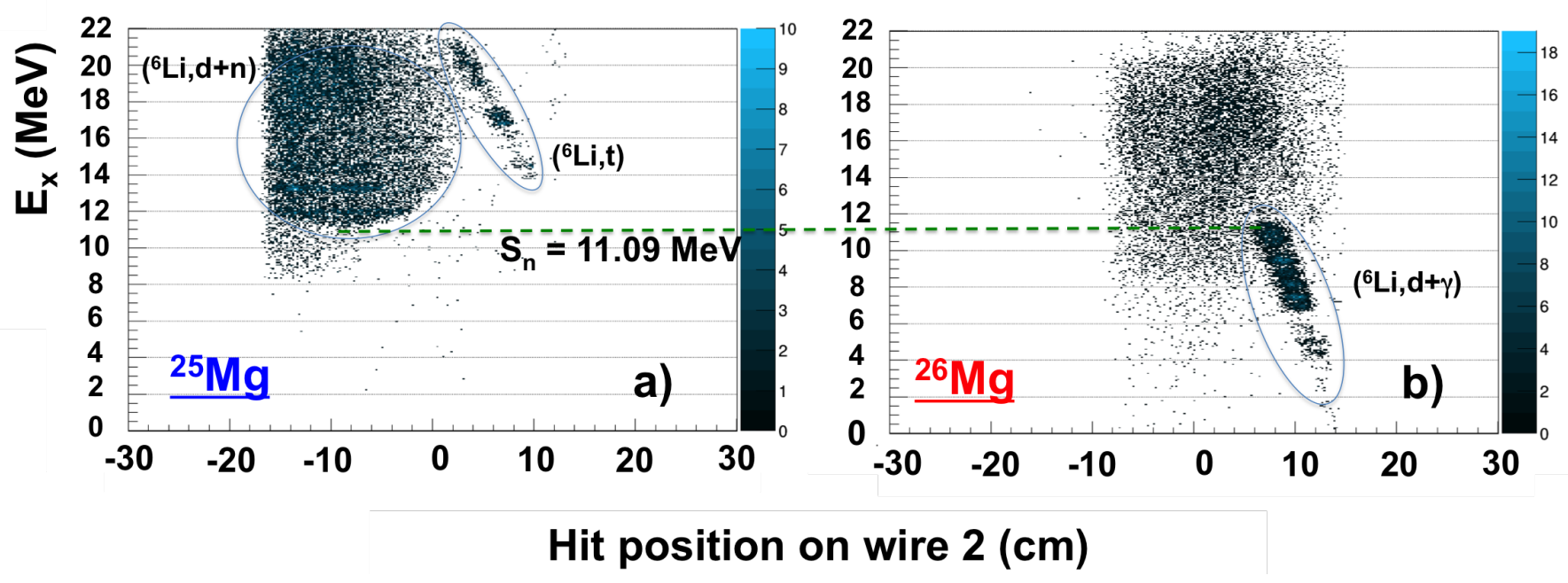

FIG. 4. $E_{x}$ versus hit position on the focal plane in the Oxford detector. All excitation energies are constructed from Hyballdetected light particle momenta, assuming the ${ }^{22} \mathrm{Ne}\left({ }^{6} \mathrm{Li}, d\right)$ reaction. Right panel: gated on ${ }^{26} \mathrm{Mg}$ recoil. Left panel: gated on ${ }^{25} \mathrm{Mg}$ recoil. Clear correlations from the binary reactions $\left({ }^{6} \mathrm{Li}, d\right)$ and $\left({ }^{6} \mathrm{Li}, t\right)$ can be observed. In the ${ }^{25} \mathrm{Mg}$ recoils, $\left({ }^{6} \mathrm{Li}, d\right)$ kinematic lines are spread in x-direction due to neutron evaporation. Transition from ${ }^{26} \mathrm{Mg}$ to ${ }^{25} \mathrm{Mg}$ is clearly occurring at the neutron separation energy of ${ }^{26} \mathrm{Mg}(11.09 \mathrm{MeV})$.

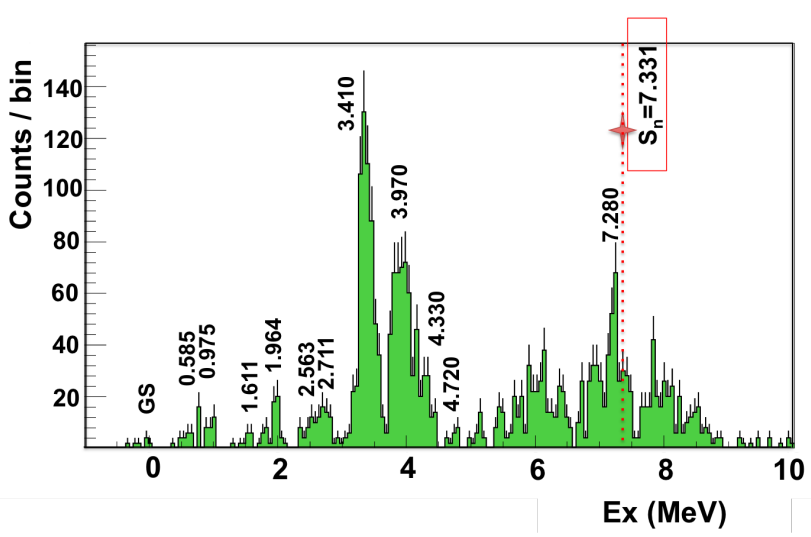

FIG. 5. ${ }^{25} \mathrm{Mg}$ excitation energy spectrum measured from the ${ }^{6} \mathrm{Li}\left({ }^{22} \mathrm{Ne}, t\right){ }^{25} \mathrm{Mg}$ reaction at $\theta_{C M}=7^{\circ}-14^{\circ}$.

all appear at energies that are close to known negative parity states, only a handful of which exist, especially in the low energy range $\left(E_{x}<5 \mathrm{MeV}\right)$ [67]. If these peaks do indeed correspond to the negative parity candidate states, this suggests a possible strong contribution from the $f p$ shell. The $E_{x}=3413,3970,4277 \mathrm{keV}$ states are rotational members of the $K=1 / 2^{-}$band $\left(\left[N n_{z} \Lambda \Omega\right]=[330\right.$ $1 / 2]$ ), which is obtained from one-particle excitation in the $f p$-shell [76-78]. It is therefore reasonable to deduce that the mechanism is similar to transfer of a neutron into the $f p$ shell in ${ }^{24} \mathrm{Mg}(d, p)$. The spectra obtained by ${ }^{24} \mathrm{Mg}(d, p \gamma)$ reactions are indeed similar to our spectra to some degree, e.g. showing highly populated $E_{x}=3413$, 3970,4277 , and $\sim 7200 \mathrm{keV}$ states $[77,79,80]$.
Concerning the other two proposed transfer mechanisms, there are currently no ${ }^{23} \mathrm{Na}(\alpha, d)$ reaction data available for comparison, and while some relevant ${ }^{22} \mathrm{Ne}(\alpha, n \gamma)$ data do exist [77, 81, 82], the associated publications do not show excitation spectra of ${ }^{25} \mathrm{Mg}$ or report specific selectivity of the reaction. As a result comparisons with $(\alpha, d)$ or $(\alpha, n)$ spectra are not currently possible.

Another point of interest is that our $\left({ }^{6} \mathrm{Li}, t\right)^{25} \mathrm{Mg}$ spectra continues beyond the neutron separation energy $\left(S_{n}=7331 \mathrm{keV}\right)$ up to $E_{x}=8.5-9 \mathrm{MeV}$, without neutron decaying into ${ }^{24} \mathrm{Mg}$ (note the spectrum is gated on ${ }^{25} \mathrm{Mg}$ ). This indicates high spin states, $I \geq(11 / 2) \hbar$ are populated since these states would have a suppressed neutron decay probability resulting from the large centrifugal barrier. The enhancement of high-spin states is to be expected due to the highly negative $Q$-value of the $\left({ }^{6} \mathrm{Li}, t\right)$ reaction $(-15.8 \mathrm{MeV})$. However, we also note that similar high spin states were observed by the ${ }^{24} \mathrm{Mg}(d, p \gamma)$ reaction [77].

Figure 6 shows the angular distribution of $E_{x}=3410$, 3973, 4330, and $7280 \mathrm{keV}$ peaks. DWBA calculations were made in the same manner as Ref. [49] using the optical potential parameters [83] in Table III. The calculations were compared with the data to extract spectroscopic factors given by $S_{\left({ }^{6} \mathrm{Li}, t\right)}=$ $(\mathrm{d} \sigma / \mathrm{d} \Omega)_{\text {exp }} /(\mathrm{d} \sigma / \mathrm{d} \Omega)_{D W B A}$, along with spin-parity assignments. The results of this analysis are shown in Table II. The cross sections and spectroscopic factors were extracted using a similar ${ }^{22} \mathrm{Ne}+{ }^{6} \mathrm{Li}$ normalization technique to the ${ }^{22} \mathrm{Ne}(d, p)$ data (Section II D), i.e. normalizing to elastic scattering cross sections in the Barrel detector. This technique produced cross sections that are only 


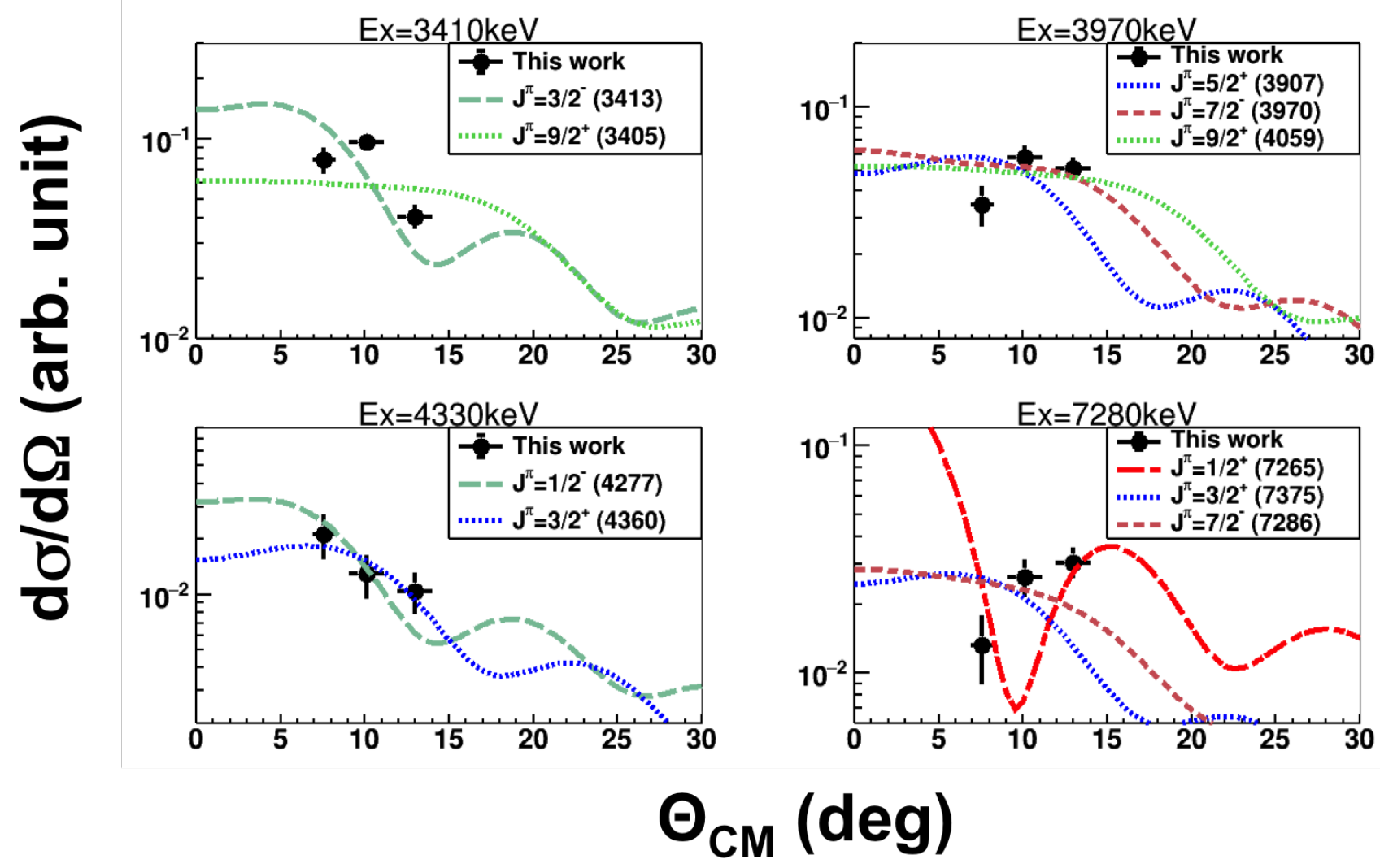

FIG. 6. Angular differential cross sections of ${ }^{22} \mathrm{Ne}\left({ }^{6} \mathrm{Li}, t\right)$ reaction for populating various states of ${ }^{25} \mathrm{Mg}$, compared with DWBA calculations. The excitation energies denoted on top of each panel are ${ }^{25} \mathrm{Mg}$ excitation energies deduced from the spectra in Fig. 5. $J^{\pi}$ and energies in the legends are from [67].

in the ballpark of expected $\left({ }^{6} \mathrm{Li}, t\right)$ cross sections because the elastic-scattering spectra unfortunately contained unknown levels of contamination from $\mathrm{F}$ and $\mathrm{C}$ components of the target. As a result, the cross sections and spectroscopic factors should be cautiously treated as being arbitrarily normalized. However, comparisons of relative spectroscopic factors between states are expected to be robust.

In the following paragraph, we discuss some of the extracted features of the most strongly observed peaks in the spectrum. Since these peaks may contain two or more unresolved states, we have used the shapes of the measured angular distributions to determine the most likely constituents, when possible. Additionally, we have used the published ${ }^{24} \mathrm{Mg}(d, p)$ data as a tentative guide to understanding the spectrum. This follows the arguments given previously that the $\left({ }^{6} \mathrm{Li}, t\right)$ reaction may populate a similar set of states as ${ }^{24} \mathrm{Mg}(d, p)$. A similar analysis was performed for the less strongly populated peaks in the spectrum, with results reported in Table II.

The $E_{x}=3410 \mathrm{keV}$ peak is likely to be dominated by the $3413 \mathrm{keV}\left(J^{\pi}=3 / 2^{-}\right)$state, as the calculated angular distribution is a much better match to the data. This is consistent with the ${ }^{24} \mathrm{Mg}(d, p)$ experiment, which also shows a much stronger transition to the $3413 \mathrm{keV}$ state, compared to the $3405 \mathrm{keV}$ state [79]. The $E_{x}=3970$ $\mathrm{keV}$ peak may come from multiple candidate states$3907 \mathrm{keV}\left(J^{\pi}=5 / 2^{+}\right), 3970 \mathrm{keV}\left(J^{\pi}=7 / 2^{-}\right)$, or $4059 \mathrm{keV}$ $\left(J^{\pi}=9 / 2^{+}\right)$. All of these candidate states have calculated angular distributions consistent with the data. For the $E_{x}=4277 \mathrm{keV}$ peak, both the $4277 \mathrm{keV}\left(J^{\pi}=1 / 2^{-}\right)$and $4360 \mathrm{keV}\left(J^{\pi}=3 / 2^{+}\right) \mathrm{keV}$ states are possibilities, having angular distributions consistent with the data. These two peaks, however, are also likely to be dominated by one single state, (3970 and $4277 \mathrm{keV}$, respectively), again following the ${ }^{24} \mathrm{Mg}(d, p)$ experiment. The $E_{x}=7280 \mathrm{keV}$ peak likely comes from the $7265 \mathrm{keV}$ state $\left(J^{\pi}=1 / 2^{+}\right)$.

Despite the difficulty of interpreting the present spectrum, the selectivity observed in detail in the $\left({ }^{6} \mathrm{Li}, t\right)$ reaction indicates that it may be a useful tool for future nuclear structure studies, e.g., as an experimental alternative to use of $(d, p)$ reaction, including experiments utilizing unstable beams in inverse kinematics. The disadvantage is that the theoretical interpretation and the direct reaction mechanism are significantly more complex. 
TABLE II. ${ }^{25} \mathrm{Mg}$ states populated in the present $\left({ }^{6} \mathrm{Li}, t\right)$ experiment. Excitation energies and spin-parities are adopted from [67]. Relative spectroscopic factors for each state, extracted from the cross-sections obtained by normalizing to the estimated beam intensity, are also listed. Note the spectroscopic factors are obtained by assuming one single state dominates in a given multiplet peak.

\begin{tabular}{ccc}
\hline \hline$E_{x}(\mathrm{keV})$ & $J^{\pi}$ & $C^{2} S_{(6 \mathrm{Li}, t)}$ \\
\hline$G S$ & $5 / 2^{+}$ & $0.02(1)$ \\
585 & $1 / 2^{+}$ & $0.04(2)$ \\
974 & $3 / 2^{+}$ & $0.11(2)$ \\
1611 & $7 / 2^{+}$ & $0.08(3)$ \\
1964 & $5 / 2^{+}$ & $0.09(2)$ \\
2563 & $1 / 2^{+}$ & $0.16(3)$ \\
2737,2801 & $7 / 2^{+}, 3 / 2^{+}$ & $0.20(4), 0.12(2)$ \\
3405,3413 & $9 / 2^{+}, 3 / 2^{-}$ & $0.41(8), 0.34(6)$ \\
$3907,3970,4059$ & $5 / 2^{+}, 7 / 2^{-} 2,9 / 2^{+}$ & $0.18(4), 0.12(3), 0.30(6)$ \\
4277,4359 & $1 / 2^{-}, 3 / 2^{+}$ & $0.14(3), 0.08(2)$ \\
4711,4722 & $9 / 2^{+},\left(3 / 2^{+}, 5 / 2^{+}\right)$ & $0.03(1), 0.03(1), 0.02(1)$ \\
$7265,7286,7375$ & $1 / 2^{+}, 7 / 2^{-}, 3 / 2^{+}$ & $0.66(17), 0.03(1), 0.09(2)$ \\
\hline \hline
\end{tabular}

TABLE III. Optical parameters used in FRESCO for DWBA analysis of ${ }^{22} \mathrm{Ne}\left({ }^{6} \mathrm{Li}, d\right){ }^{26} \mathrm{Mg}[49]$ and ${ }^{22} \mathrm{Ne}\left({ }^{6} \mathrm{Li}, t\right){ }^{25} \mathrm{Mg}$ [83]. All radii except those for the $\alpha+d$ channel are given such that $R_{x}=r_{x} A^{1 / 3}$. For the $\alpha+d$ channel, $R_{x}=r_{x}$.

\begin{tabular}{|c|c|c|c|c|c|c|c|c|c|c|c|c|c|}
\hline Channel & $\begin{array}{c}r_{c} \\
(\mathrm{fm})\end{array}$ & $\begin{array}{c}V_{r} \\
(\mathrm{MeV})\end{array}$ & $\begin{array}{c}r_{r} \\
(\mathrm{fm})\end{array}$ & $\begin{array}{c}a_{r} \\
(\mathrm{fm})\end{array}$ & $\begin{array}{c}W_{i} \\
(\mathrm{MeV})\end{array}$ & $\begin{array}{c}r_{i} \\
(\mathrm{fm})\end{array}$ & $\begin{array}{c}a_{i} \\
(\mathrm{fm})\end{array}$ & $\begin{array}{c}W_{D} \\
(\mathrm{MeV})\end{array}$ & $\begin{array}{c}r_{D} \\
(\mathrm{fm})\end{array}$ & $\begin{array}{c}a_{D} \\
(\mathrm{fm})\end{array}$ & $\begin{array}{c}V_{s o} \\
(\mathrm{MeV})\end{array}$ & $\begin{array}{l}r_{s o} \\
(\mathrm{fm})\end{array}$ & $\begin{array}{l}a_{\text {so }} \\
(\mathrm{fm})\end{array}$ \\
\hline${ }^{22} \mathrm{Ne}+{ }^{6} \mathrm{Li}$ & 1.30 & 117.04 & 1.80 & 0.40 & 48.60 & 1.99 & 0.62 & & & & & & \\
\hline${ }^{26} \mathrm{Mg}+d$ & 1.30 & 79.07 & 1.17 & 0.79 & 2.99 & 1.33 & 0.74 & 10.51 & 1.33 & 0.74 & 5.88 & 1.07 & 0.66 \\
\hline$\alpha+d$ & 1.90 & $\mathrm{a}$ & 1.90 & 0.65 & & & & & & & & & \\
\hline Final State & 1.40 & $\mathrm{~b}$ & 1.40 & 0.70 & & & & & & & & & \\
\hline${ }^{25} \mathrm{Mg}+t$ & 1.42 & 149.57 & 1.07 & 0.74 & 9.65 & 1.26 & 1.18 & 31.95 & 1.09 & 0.85 & 1.90 & 0.51 & 0.20 \\
\hline${ }^{3} \mathrm{He}+t$ & 1.25 & a & 1.25 & 0.65 & & & & & & & & & \\
\hline Final State & 1.40 & c & 1.40 & 0.70 & & & & & & & & & \\
\hline
\end{tabular}

${ }^{\text {a }}$ Adjusted to give the correct ${ }^{6} \mathrm{Li}$ binding energy

b Adjusted to give the correct final state binding/resonance energy

c Adjusted to give the correct final state binding energy

\section{IV. ${ }^{22} \mathbf{N E}\left({ }^{6} \mathbf{L I}, d\right){ }^{26} \mathrm{MG}$ REACTION}

Figure 7 shows the ${ }^{26} \mathrm{Mg}$ excitation spectrum obtained from the ${ }^{22} \mathrm{Ne}\left({ }^{6} \mathrm{Li}, d\right){ }^{26} \mathrm{Mg}$ reaction in the present experiment (the two spectra gated on either ${ }^{26} \mathrm{Mg}$ or ${ }^{25} \mathrm{Mg}$ were summed.) A number of resolved and unresolved peaks are evident. In the following sections, the states populated in the reaction are identified and analyzed using the angular distributions of the deuterons, along with the coincident $\gamma$ rays detected in the HPGe detectors. We also compare our results with those of past (normal kinematics) studies: Refs. [40, 42, 84, 85], which utilized ${ }^{6} \mathrm{Li}$ beams with energies of 5.3 [84], 5.3 [40], 5.0 [85], and $13.5 \mathrm{MeV} /$ nucleon [42], respectively. The previous studies varied in the range of excitation energies to which they were sensitive: $0-9 \mathrm{MeV}$ (but with peaks identified and analyzed only up to $5.7 \mathrm{MeV}$; We read the higher $E_{x}$ by digitizing their spectrum.) [84], 9.3-12 $\mathrm{MeV}$ [40], 9-11 MeV [85], and 7.3-11.4 MeV [42]. The present experiment is sensitive to $E_{x}>4 \mathrm{MeV}$. Below $E_{x}=4 \mathrm{MeV}$, the ejected deuterons punch through the Hyball, and ${ }^{26} \mathrm{Mg}$ recoils are also outside of the Oxford detector acceptance window. The assumed states thus identified are annotated in Figure 7. Because both $\alpha$ particles and ${ }^{22} \mathrm{Ne}$ have $0^{+}$spins, natural parity states such as $J^{\pi}=0^{+}, 1^{-}$, and $2^{+}$are selectively populated in our experiment. Moreover, because of the selectivity for $\alpha$-cluster states by $\alpha$-transfer reactions, we assigned these states based on the states observed in the past $\left({ }^{6} \mathrm{Li}, d\right)$ studies. The states observed in the past $\left({ }^{6} \mathrm{Li}, d\right)$ studies were summarized in Table IV.

Figure 8 shows $\gamma$-ray spectra in coincidence with ${ }^{25,26} \mathrm{Mg}+d$, as well as the spectrum of $\gamma$-rays in coincidence with ${ }^{23} \mathrm{Ne}+d$ from the ${ }^{22} \mathrm{Ne}(d, p)^{23} \mathrm{Ne}$ measurement (Section IID). The high statistics of the latter spectrum make it a useful guide in interpreting the $\gamma$ rays from the ${ }^{22} \mathrm{Ne}\left({ }^{6} \mathrm{Li}, d \gamma\right)$ measurement. Before discussing the $\gamma$ ray spectra in coincidence with specific excitation energy regions, we briefly discuss some features of the total spectrum here.

In the ${ }^{26} \mathrm{Mg}$ coincidence data, two large peaks (first excited state: 1808 $\rightarrow$ GS) and (second excited state: 2938 $\rightarrow 1808$ ) are evident. This is to be expected as these transitions are fed by a large num- 
TABLE IV. ${ }^{26} \mathrm{Mg}$ states populated in the past $\left({ }^{6} \mathrm{Li}, d\right)[40,42,84,85]$ and $(\alpha, n)[36]$ experiments. Excitation energies in the first column were taken from [67] $(<7.365 \mathrm{MeV})$ and [42]. Excitation energies above $5715 \mathrm{keV}$ by [84] were extracted by digitizing their spectrum (Fig. 3 in their work). Note the uncertainties $\left({ }_{-0}^{+180} \mathrm{keV}\right)$ are evaluated from our scanning processes. For the states between $10000 \mathrm{keV}$ and $11800 \mathrm{keV}$, see [49].

\begin{tabular}{|c|c|c|c|c|c|}
\hline$E_{x}(\mathrm{keV})$ (candidate states) & {$[84]$} & {$[40]$} & [85] & {$[42]$} & {$[36]$} \\
\hline 4318,4332 & $4320,4330\left(4228_{-0}^{+180 a}\right)$ & & & & \\
\hline $4835,4901,4970$ & $4840,4900,4970\left(4797_{-0}^{+180 a}\right)$ & & & & \\
\hline 5476 & $5470\left(5329_{-0}^{+180 \mathrm{a}}\right)^{-0}$ & & & & \\
\hline 5715 & $5690\left(5611_{-0}^{+180 a}\right)$ & & & & \\
\hline 6622,6634 & $6475_{-0}^{+180}$ & & & & \\
\hline 6756,6876 & $6741_{-0}^{+180}$ & & & & \\
\hline $6952,6972,6978,7061,7099$ & $6929_{-0}^{+180}$ & & & & \\
\hline $7365(13)$ & $7235_{-0}^{+180}$ & & & $7365(13)$ & \\
\hline $7685(8)$ & $7562_{-0}^{+180}$ & & & $7671(16)$ & \\
\hline $7826(6)$ & $7695_{-0}^{+180}$ & & & $7821(22)$ & \\
\hline $8036(7)$ & $7916_{-0}^{+180}$ & & & $8040(13)$ & \\
\hline $8193(15)$ & $8101_{-0}^{+180}$ & & & $8214(14)$ & \\
\hline $8626(7)$ & $8550_{-0}^{+180}$ & & & $8625(15)$ & \\
\hline $8937(6)$ & $8868_{-0}^{+180}$ & & & $8931(13)$ & \\
\hline $9383(16)$ & $9250_{-0}^{+180}$ & $9404(20)$ & $9320(60)$ & $9383(16)$ & \\
\hline $9603(9)$ & & $9586(20)$ & $9570(40)$ & $9595(32)$ & \\
\hline $9992(8)$ & & $9985(20)$ & & $9987(18)$ & \\
\hline $11824(9)$ & & $11831(20)$ & & & $11828(2)$ \\
\hline
\end{tabular}

${ }^{a}$ Obtained by digitizing the spectrum from [84] and shown for comparison with the energies they reported.

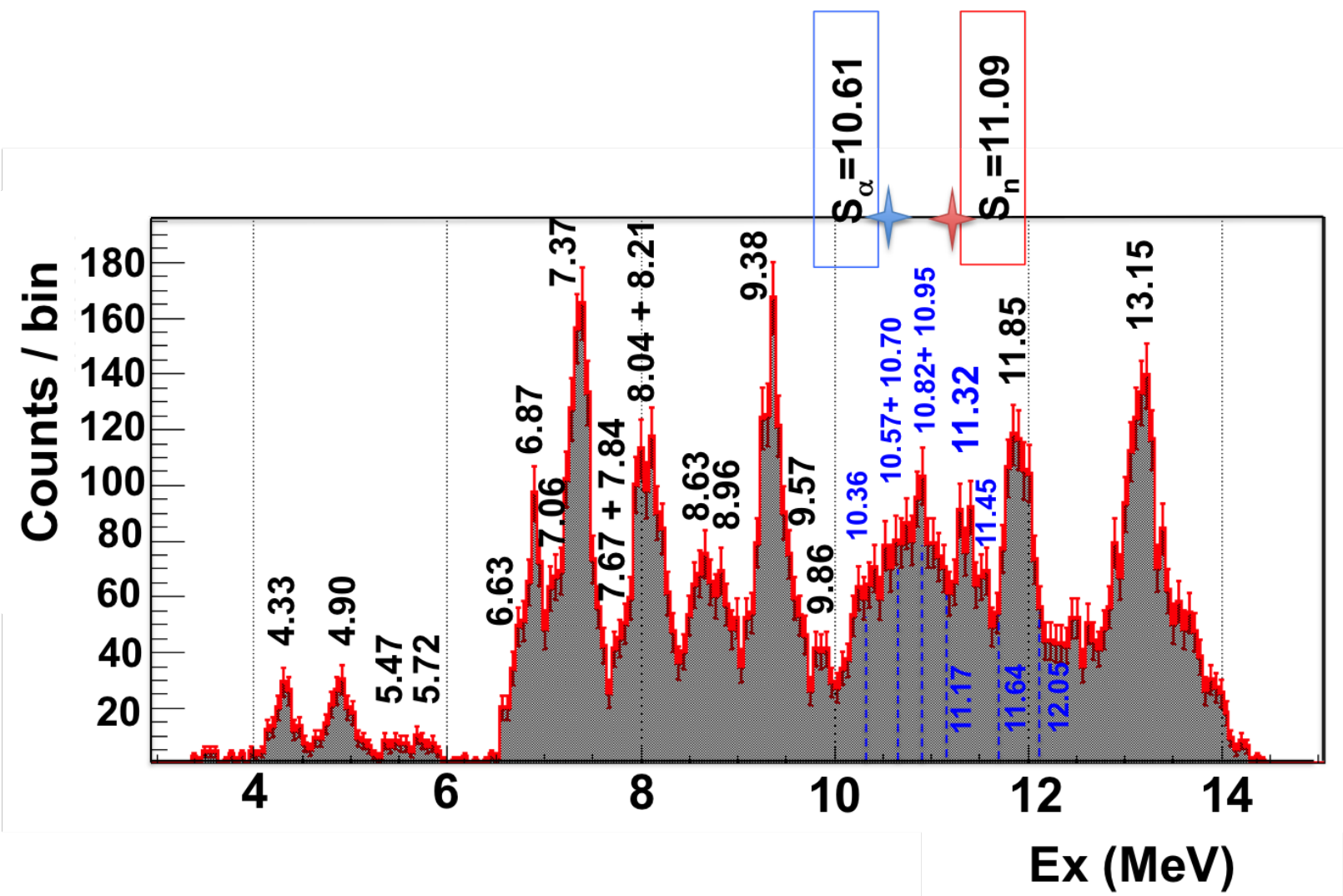

FIG. 7. ${ }^{26} \mathrm{Mg}$ excitation energy spectrum measured from the ${ }^{6} \mathrm{Li}\left({ }^{22} \mathrm{Ne}, d\right){ }^{26} \mathrm{Mg}$ reaction at $\theta_{C M}=7^{\circ}-14^{\circ}$. All states considered in the present data analysis are labeled in the figure. These states are mostly determined with the help of the coincident $\gamma$ rays and past $\left({ }^{6} \mathrm{Li}, d\right)$ experiments. The energies shown are adopted by comparing our measured energies with Table IV. 
ber of higher-lying states. Additionally, small peaks $(4318 / 4332 / 4350 \rightarrow 1808)$ and $(3941 \rightarrow 2938)$ are present. These smaller peaks are more easily observed when we gate on a specific excitation energy from the deuteron spectrum, as discussed in the subsequent sections. In addition to the prominent peaks, we also observe a background from Compton scattering (our HPGe detectors are not Compton-suppressed). To better understand this, we used the high-statistics ${ }^{22} \mathrm{Ne}(d, p \gamma)$ data (Figure $8 \mathrm{a}$ ) to estimate the ratio of the Compton edges of the well-separated 1016 and $2203 \mathrm{keV}$ transitions to their photopeaks (peak-to-peak ratio). This analysis suggests $\mathrm{a} \sim 15-20 \%$ ratio for $1-2 \mathrm{MeV} \gamma$-rays (also confirmed with the Geant 4 simulations).

In the bottom panel of Figure 8, we show the $\gamma$-ray spectrum in coincidence with ${ }^{25} \mathrm{Mg}$ recoils and deuterons in the $11-14 \mathrm{MeV}$ excitation energy range of ${ }^{26} \mathrm{Mg}$. The observed $\gamma$ rays correspond to transitions in ${ }^{25} \mathrm{Mg}$ following neutron evaporation of states in ${ }^{26} \mathrm{Mg}$. We observe $384,585,974,1379,1611 \mathrm{keV} \gamma$-ray transitions from the first, second, and third excited states of ${ }^{25} \mathrm{Mg}$, suggesting that states in this region have a significant neutron decay branch to low-lying excited ${ }^{25} \mathrm{Mg}$ states. This is consistent with Ref. [34], which reported significant $\left(\alpha, n_{1}\right)$ and $\left(\alpha, n_{2}\right)$ strengths for a number of states observed in a direct ${ }^{22} \mathrm{Ne}(\alpha, n \gamma){ }^{25} \mathrm{Mg}$ experiment. Since we do not measure neutrons, it is not realistic to estimate the populated ${ }^{26} \mathrm{Mg}$ states from the $\gamma$-ray transitions alone. However, limited information about the decay properties of ${ }^{26} \mathrm{Mg}$ states in this region can still be obtained from the coincident $\gamma$-ray analysis, as discussed in Section IV A 13.

After identifying the set of populated states, using particle- $\gamma$ coincidences with the help of past $\left({ }^{6} \mathrm{Li}, d\right)$ data (see Table IV), we performed a multiple Gaussian fit to peaks in the deuteron-reconstructed excitation energy spectrum. These fits were then used to determine cross sections and angular distributions of a given peak. For the fits, the centroid of each Gaussian was taken to be the adopted energy of the corresponding state (shown in Figure 7), and the width of each Gaussian was set according to the experimental energy resolution (FWHM 200-250 keV, depending on excitation energy). Amplitudes were allowed to freely vary, and the resulting Gaussian integrals were used to determine a differential cross section for each state. The resulting angular distributions for each of the noticable peaks in the deuteron spectrum are displayed in Figure 9. Using these distributions, spin-parities and relative spectroscopic factors were determined, as presented in Table $\mathrm{V}$ and discussed in Section IV B.

\section{A. Populated states}

Figure 10 shows $\gamma$-ray spectra in coincidence with specific ${ }^{26} \mathrm{Mg}$ total excitation energy ranges. Simple Monte Carlo simulations of $\gamma$ spectra from the respective states were also made to estimate the expected counts of respec-
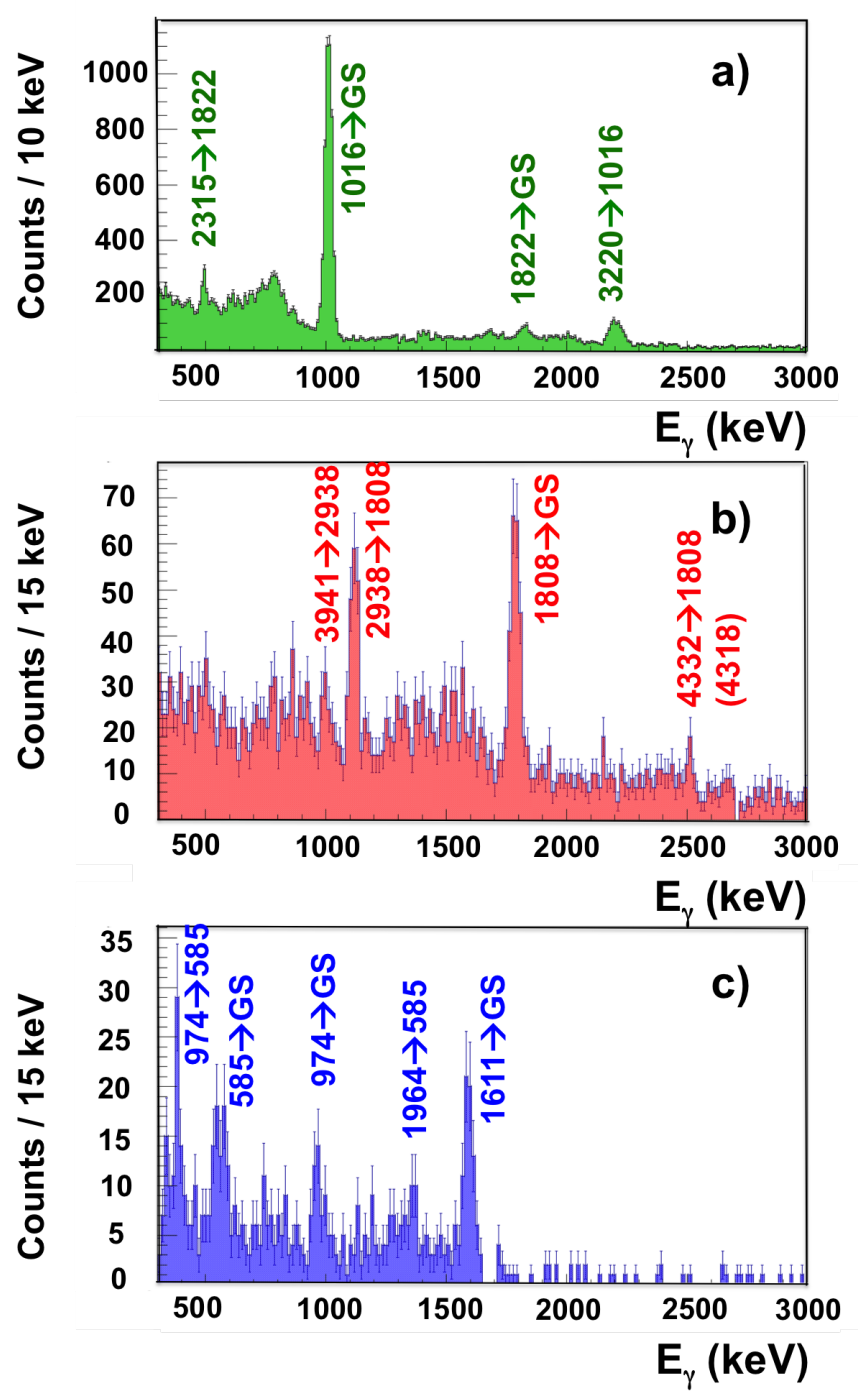

FIG. 8. Panel (a): $\gamma$-ray spectrum from ${ }^{22} \mathrm{Ne}(d, p \gamma)^{23} \mathrm{Ne}$ reaction in coincidence with $E_{x}=\mathrm{GS}$ to $5 \mathrm{MeV}$ from the proton excitation-energy spectrum. Panel (b): $\gamma$-ray spectrum from the ${ }^{22} \mathrm{Ne}\left({ }^{6} \mathrm{Li}, d \gamma\right){ }^{26} \mathrm{Mg}$ reaction in coincidence with $E_{x}=4-11.5$ $\mathrm{MeV}$. Panel (c): $\gamma$-ray spectrum from the ${ }^{22} \mathrm{Ne}\left({ }^{6} \mathrm{Li}, d n \gamma\right){ }^{25} \mathrm{Mg}$ reaction in coincidence with $E_{x}\left({ }^{26} \mathrm{Mg}\right)=11.0-14.0 \mathrm{MeV}$

deuterons.

tive $\gamma$-ray transitions, with the level table [67] and our HPGe detector efficiency as inputs. Background counts by random coincidence were estimated from events located near the photopeaks of interest.

$$
\text { 1. } E_{x}=4.0-4.6 \mathrm{MeV}
$$

Possible states making up the peak in this region are $4.318\left(4^{+}\right)$or $4.332 \mathrm{MeV}\left(2^{+}\right)$. Since the $4.350 \mathrm{MeV}\left(3^{+}\right)$ state is an unnatural parity state, it can be excluded. The possible $\gamma$ transitions from states in this region are therefore $(4318 \rightarrow 1808 \rightarrow$ G.S. $)(99 \%)$ and $(4332 \rightarrow 1808 \rightarrow$ G.S. $)$ 

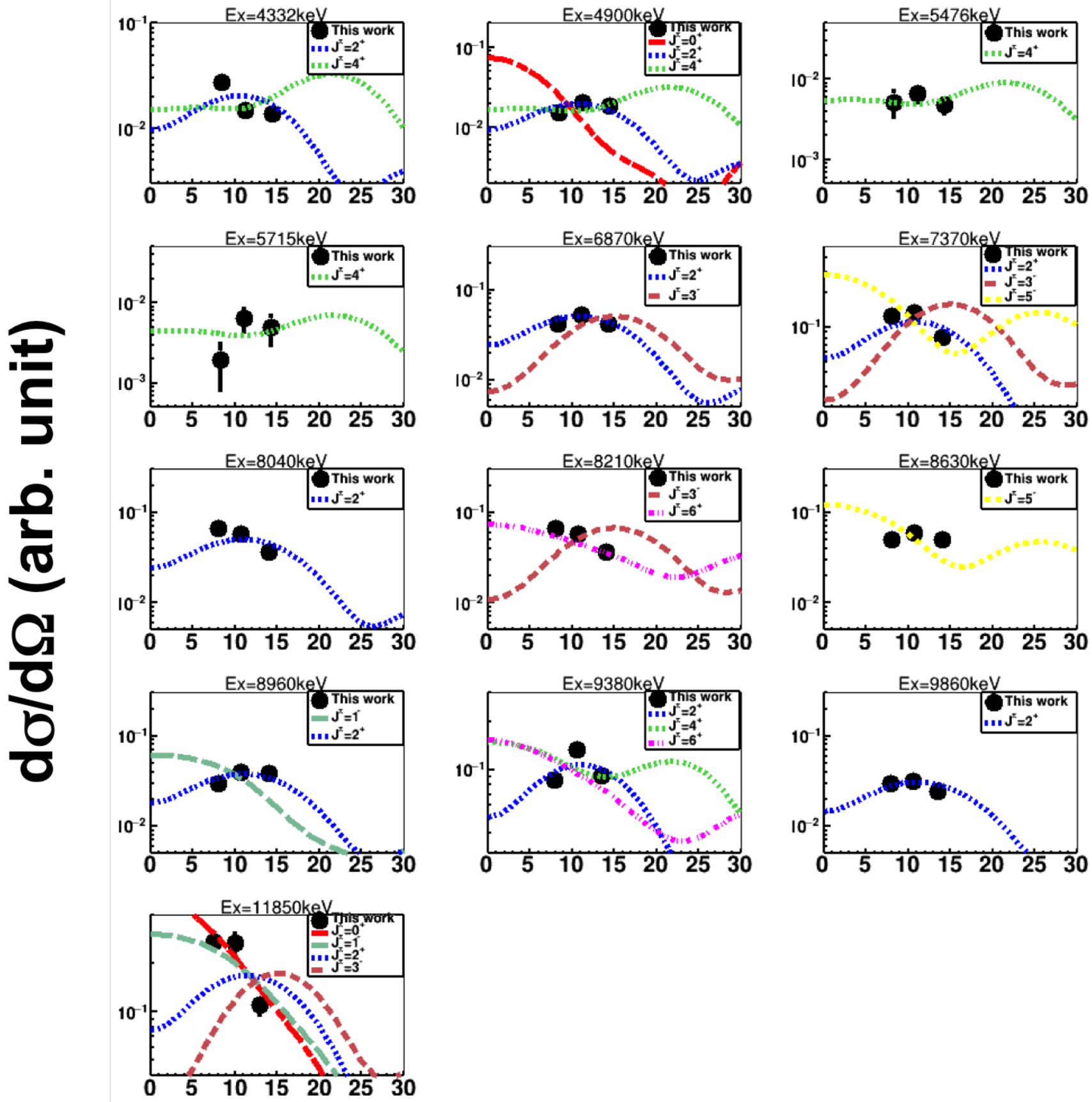

\section{$\Theta_{\mathrm{CM}}(\mathrm{deg})$}

FIG. 9. Angular differential cross sections of ${ }^{22} \mathrm{Ne}\left({ }^{6} \mathrm{Li}, d\right)$ reactions for populating various states of ${ }^{26} \mathrm{Mg}$, compared with DWBA calculations.

$(79 \%) /(4332 \rightarrow 2938 \rightarrow 1808 \rightarrow$ G.S. $)(15 \%)$, where percentages in parenthesis denote the $\gamma$-decay branching ratios from the state. Since there seems to be the 1394 $\mathrm{keV} \gamma$ transition from $4332 \rightarrow 2938 \mathrm{keV}$, as well as the subsequent $1129 \mathrm{keV}$ transition from $2938 \rightarrow 1808 \mathrm{keV}$, it is most likely that the peak exclusively consists of the
$4332 \mathrm{keV}$ state. There are $\sim 150$ total deuteron events in the peak, and these two coincident $\gamma$-ray transitions both contain 3-5 counts after background subtraction. These rates are slightly higher than, but statistically consistent with, the expected 1-2 counts in these peaks after accounting for branching ratios and detection efficiencies. 


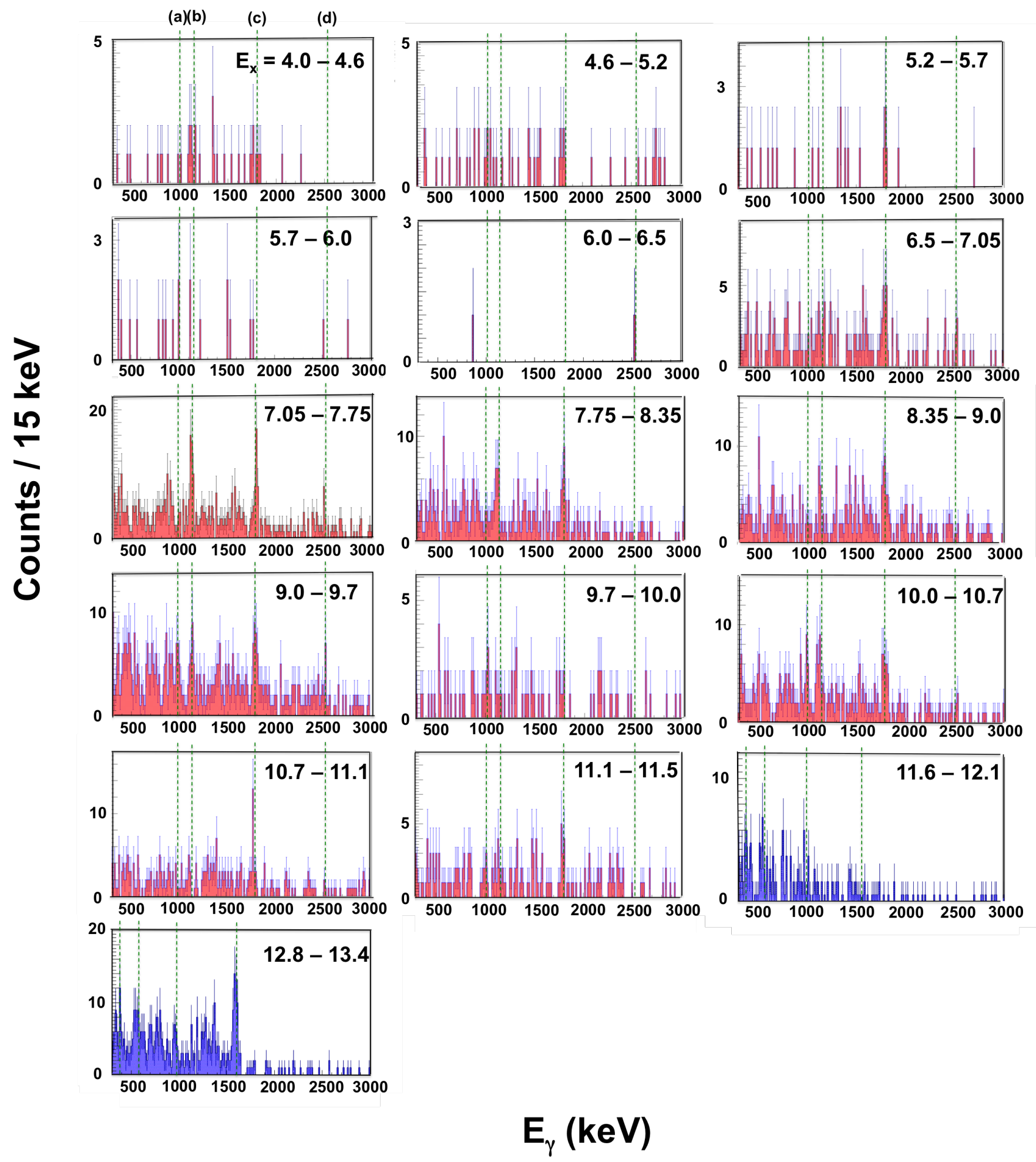

FIG. 10. Coincident $\left({ }^{6} \mathrm{Li}, d\right) \gamma$-ray spectra, gated on specific excitation energy ranges in ${ }^{26} \mathrm{Mg}$. Note the last two panels (the blue-shaded histogram) were obtained by gating on ${ }^{25} \mathrm{Mg}$ recoils instead of ${ }^{26} \mathrm{Mg}$. The vertical dotted lines indicate the energies of major transitions from the low-lying states in ${ }^{26} \mathrm{Mg}$ : (a) 1003, (b) 1129, (c) 1808, and (d) 2510/2523 keV, respectively (see Figure 8 (b)). The vertical lines indicate 389, 585, 974, and $1611 \mathrm{keV}$ transitions (major transitions from the low-lying states in ${ }^{25} \mathrm{Mg}$; see Figure 8 (c)) in the last two panels. 
From both states, 3-5 counts of another major transition $(2510 / 2523 \mathrm{keV})$ are expected. No such transitions are, however, observed. Therefore, it is not possible to determine the state unambiguously in the present study. The assignment of the $4.332 \mathrm{MeV}$ state is supported by the angular distribution, which is consistent with a $2^{+}$ DWBA calculation (see Figure 9).

\section{2. $E_{x}=4.6-5.2 \mathrm{MeV}$}

There is an evident peak around $E_{x}=4.901 \mathrm{MeV}\left(4^{+}\right)$, which could be contaminated by $E_{x}=4.835 \mathrm{MeV}\left(2^{+}\right)$ or $4.972 \mathrm{MeV}\left(0^{+}\right)$. Figure 10 shows the coincident $\gamma$ spectrum. While 4.835 and $4.970 \mathrm{MeV}$ states have the major $1129 \mathrm{keV}$ transition $(2938 \rightarrow 1808)$ with expected 6-7 counts, this line is not present in the $\gamma$-ray spectrum. Therefore, the $4.901 \mathrm{MeV}$ state is likely the main contributor to the peak. This is supported by our angular distribution, which is consistent with $2^{+}$or $4^{+}$calculations. We note that the $1808 \mathrm{keV} \gamma$-transition $(1808 \rightarrow$ GS) is expected from any of the candidate states with $\sim 100 \%$ probability. The observed number of $1808 \mathrm{keV}$ events ( $\sim 5$ counts) is consistent with the expectations for this peak (5-6 counts for any of the states).

\section{3. $E_{x}=5.2-6.0 \mathrm{MeV}$}

There is a small peak on the left side of this region, which we assign to the $E_{x}=5.476 \mathrm{MeV}\left(4^{+}\right)$state reported by Ref. [84]. A few counts of the $1808 \mathrm{keV}$ transition are expected as evidence for the population of this state and observed in the $\gamma$-ray spectrum. The angular distribution is also consistent with the $4^{+}$calculation.

Another small peak (on the right side of this region) is assigned to the $5.715 \mathrm{MeV}$ state $\left(4^{+}\right)$, also reported by Ref. [84]. For this peak, no decisive $\gamma$-ray transition was observed due to poor statistics. Our measured angular distribution agrees poorly with the $4^{+}$calculation; however, the same inconsistency is observed in Ref. [84], where it is attributed to possible multi-step reaction contributions.

$$
\text { 4. } E_{x}=6.0-7.0 \mathrm{MeV}
$$

There are no states populated between $E_{x}=6.0-6.5$ $\mathrm{MeV}$, which agrees well with Ref. [84]. A coincident 1808 $\mathrm{keV} \gamma$-ray transition is clearly seen in coincidence with the excitation range of $6.5-7 \mathrm{MeV}$. This supports population of the $E_{x}=6.876 \mathrm{MeV}\left(3^{-}\right)$state, which dominantly decays via the $1808 \mathrm{keV}$ transition. There is another candidate state, the $6.745 \mathrm{MeV}\left(2^{+}\right)$. The state, however, should have the comparable number of $1129 \mathrm{keV}$ transition to the $1808 \mathrm{keV}$ transition. Therefore, the peak more likely corresponds to the $6.876 \mathrm{MeV}$ state. This assignment is further supported by agreement of the angular distribution with the $3^{-}$calculations, although it slightly better agrees with the $2^{+}$. It is also worth mentioning that Ref. [84] observed states at 6.63 and 7.06 $\mathrm{MeV}$ as well, but with much weaker intensity than the 6.876 MeV. Evidence for a $6.63 \mathrm{MeV}$ shoulder is present in the deuteron spectrum, although no clear $\gamma$ rays from this state are observed. We also note that there appears to be a $\gamma$-ray transition around $1600 \mathrm{keV}$ in coincidence with this excitation region, even though no known states populated by $\left({ }^{6} \mathrm{Li}, d\right)$ reactions are expected to generate this $\gamma$-ray $[67,86]$.

$$
\text { 5. } E_{x}=7.0-7.7 \mathrm{MeV}
$$

The large peak observed in this region by this and other $\left({ }^{6} \mathrm{Li}, d\right)$ experiments $[42,84]$ could arise from any of the $7.348\left(3^{-}\right), 7.371\left(2^{+}\right)$, or $7.396 \mathrm{keV}\left(\left(5^{+}\right)\right)$states [67]. The coincident $\gamma$-ray spectrum shows two strong transitions at 1808 and $1129 \mathrm{keV}$ (i.e., decay through the $E_{x}=2.938 \mathrm{MeV}$ state), with another peak at 2510 $\mathrm{keV}$. The 7.371 and $7.396 \mathrm{MeV}$ states have dominant $\gamma$-ray transitions at $1003 \mathrm{keV}$ and $1680 \mathrm{keV}$ (expected $\sim 11$ and 17 counts each), respectively. Neither of these transitions are observed, leading to the conclusion that the peak is dominated by the $7.348 \mathrm{MeV}$ state $\left(3^{-}\right)$, together with the consistency between observed and expected $1129,1808 \mathrm{keV}$, and $2510 \mathrm{keV}$ counts for this state $(\sim 30, \sim 30$, and $\sim 7$ counts, respectively). However, the observed angular distribution is not consistent with the $3^{-}$calculation. It is consistent with $2^{+}$, which agrees with Talwar et al. Therefore, an unambiguous assignment is not possible in this region.

$$
\text { 6. } E_{x}=7.7-8.3 \mathrm{MeV}
$$

There is a slightly broad peak in the energy region, which is probably a doublet consisting of the $8.034\left(2^{+}\right)$ and $8.184\left(3^{-}\right) / 8.201 \mathrm{MeV}\left(6^{+}\right)$states seen by Anantaraman et al. [84] and Talwar et al. [42]. The observed angular distribution supports both $2^{+}$and $6^{+}$assignments, excluding the $8.184 \mathrm{MeV}$ state. Moreover, the $\gamma$-ray spectrum indicates that the $8.034 \mathrm{MeV}$ state is dominant. Two $\gamma$-ray transitions are strongly observed: 1129 and $1808 \mathrm{keV}$, and additionally there is evidence for a 511 $\mathrm{keV}$ transition. The presence of the $511 \mathrm{keV}$ line would be expected from the high-energy $(8034 \rightarrow 2938)$ transition from the $8.034 \mathrm{MeV}$ state. At the same time, the lack of a $2510 \mathrm{keV}$ transition suggests an absence of the 8.201 MeV state, which is expected to give a $2510 \mathrm{keV}$ line with $\sim 50 \%$ the intensity of the $1808 \mathrm{keV}$ line. Similarly, the roughly equal 1129 and $1808 \mathrm{keV}$ intensities observed in the spectrum are not consistent with decay from either the 8.184 or $8.201 \mathrm{MeV}$ state, which is expected to produce the $1129 \mathrm{keV}$ line with only $30-40 \%$ as many counts as the $1808 \mathrm{keV}$ transition. 
The absence of the $8.184 / 8.201 \mathrm{MeV}$ state is inconsistent with the results of Talwar et al..; however, this may be due to the higher beam energy used in that study, which increases population of high-spin states. The angular distributions support both $E_{x}=8.034\left(2^{+}\right)$and 8.201 $\mathrm{MeV}\left(6^{+}\right)$states.

\section{7. $E_{x}=8.3-9.0 \mathrm{MeV}$}

There are two peaks at $E_{x}=8.63$ and $8.93 \mathrm{MeV}$ observed by Talwar et al. [42], and seemingly Anantaraman et al. [84] as well. The $\gamma$-ray spectrum shows some possible transitions, including the 1129 and $1808 \mathrm{keV}$. Presence of the $E_{x}=8625 \mathrm{keV}$ state $\left(5^{-}\right)$and $8931 \mathrm{keV}$ state $\left(1^{-}\right.$or $\left.2^{+}\right)$both explain these transitions well. The observed angular distributions are reasonably consistent with both the $2^{+}$and $5^{-}$calculations. As a result, it is likely that both states are populated although we are not sensitive to the difference between these two possible states.

\section{8. $E_{x}=9.0-9.7 \mathrm{MeV}$}

In this region there is a strong peak observed by Talwar et al. [42], Giesen et al. [40], and Ugalde et al. [85]. Possible states in this region are 9.325 (Ugalde), 9.371, and 9.383 (Talwar) MeV. Looking at the $\gamma$-ray spectrum, the number of observed $1129 \mathrm{keV}$ and $1808 \mathrm{keV}$ counts are almost the same. Hence, it is probable that a large fraction of $\gamma$ transitions occur via the $2938 \mathrm{keV}$ state. There is also a noticeable transition from either the 2510 or $2523 \mathrm{keV} \gamma$ ray, which indicates decay via the 4318 or $4331 \mathrm{keV}$ state $(\rightarrow 1808$ or $\rightarrow 2938 \rightarrow 1808)$.

If the $\left({ }^{6} \mathrm{Li}, d\right)$ peak is dominated by the $9.371 \mathrm{MeV}$ state, a $1003 \mathrm{keV} \gamma$-ray transition $(3941 \rightarrow 2938)$ is expected to be observed, with similar intensity to the 1129 $\mathrm{keV}$ and $1808 \mathrm{keV}$ transitions. This is seen in the spectrum; thus it is possible the $9.371 \mathrm{MeV}$ state is a significant contributor to the peak. If the peak is dominated by the $E_{x}=9.325 \mathrm{MeV}$ state, the $2510 / 2523 \mathrm{keV} \gamma$-ray transition is expected with fewer counts than a dominant $E_{x}=9.383 \mathrm{MeV}$ state (expected $\sim 5$ and $\sim 10$ counts, respectively). However, due to the low numbers of expected and observed counts, we cannot make a definitive distinction between these two states on this basis.

Turning to the angular distributions, the observed shape can be better explained by a $2^{+}$assignment. This tentatively favors a dominant $9.325 \mathrm{MeV}$ state, which is reported to have spins in the ranging from $2^{+}$to $4^{+}$[67]. Conversely, the $9.383 \mathrm{MeV}$ state claimed by Talwar et al. was reported having $J^{\pi}=0^{+}$or $1^{-}[42]$, or $6^{+}$[67], and the $9.371 \mathrm{MeV}$ state was reported having $4^{+}$[67], neither of the two states (although $0^{+}$and $1^{-}$are not shown in the figure) is consistent with the present data.

\section{9. $E_{x}=9.7-10.0 \mathrm{MeV}$}

While Talwar et al. and Giesen et al. both report a state at $E_{x}=9.99 \mathrm{MeV}$, we instead observed a peak at $9.856 \mathrm{MeV}$. The angular distribution supports a $2^{+}$ assignment, which agrees with our assignment to the $2^{+}$, $9.856 \mathrm{MeV}$ state [67]. No clear $\gamma$-ray transitions were observed in this region.

$$
\text { 10. } E_{x}=10-10.7 \mathrm{MeV}
$$

In this excitation region, clear $\gamma$-ray peaks are observed at 1003,1129 , and $1808 \mathrm{keV}$, in addition to a possible peak around $1550 \mathrm{keV}$. The states observed in past $\left({ }^{6} \mathrm{Li}, d\right)$ measurements are $10.36,10.57,10.70 \mathrm{MeV}$ [40, 42] (note that Talwar et al. [42] did not observe the $10.57 \mathrm{MeV}$ state). For the $10.57 \mathrm{MeV}$ state $\left(1^{-}\right)$, reliable $\gamma$-ray transition data are also available from a $\left(\gamma, \gamma^{\prime}\right)$ experiment [33]. Possible candidate states around the $10.36 \mathrm{MeV}$ region are $10.349,10.362$, and 10.377 $\mathrm{MeV}$ [67]. The $10.349 \mathrm{MeV}$ state exclusively generates a $1808 \mathrm{keV} \gamma$-ray. The $10.362 \mathrm{MeV}$ state also generates a $\sim 100 \% 1808 \mathrm{keV} \gamma$ ray via the $10362 \rightarrow 1808 \rightarrow$ GS scheme with some weaker schemes, which is several times more intense than the next strongest transition $(1129 \mathrm{keV})$. The $10.377 \mathrm{MeV}$ state can emit a 1003 $\mathrm{keV} \gamma$-ray with some strength $(\sim 25 \%)$ after transitioning through the $5.715 \mathrm{MeV}$ state $(5.715 \rightarrow 4.350 \rightarrow 1.808 \rightarrow \mathrm{GS}$ or $5.715 \rightarrow 3.941 \rightarrow 2.938 \rightarrow 1.808 \rightarrow$ GS). Hence the 10.362 and $10.377 \mathrm{MeV}$ states are reasonably consistent with the observed $\gamma$-ray spectrum.

The $10.57 \mathrm{MeV}$ state reported by Longland et al. [33] decays through the $4.972 \mathrm{MeV}$ state, generating 1129, $1808,2003 \mathrm{keV} \gamma$ rays. For this decay scheme, the 2003 $\mathrm{keV}$ transition is expected to have nearly half the intensity of the $1808 \mathrm{keV}$ transition. We do not observe a $2003 \mathrm{keV}$ transition at all, making it likely that the 10.57 MeV state is only weakly populated. This agrees with both Giesen et al. [40] and Talwar et al. [42].

The counts around $10.70 \mathrm{MeV}$ can arise from two possible candidate states. The first is at $10.707 \mathrm{MeV}$ and decays via the $5.715 \mathrm{MeV}$ state. The second is at $10.693 \mathrm{MeV}$ that also decays through the 5.715 $(10.693 \rightarrow 7.396 \rightarrow 5.715)$. Thus, the two states generate similar $\gamma$-ray spectra, except for the $1680 \mathrm{keV}$ transition from $(7.396 \rightarrow 5.715)$ in the $10.693 \mathrm{MeV}$ state. Both states generate a 1129 and $1003 \mathrm{keV}$ transition (via the 2.938 and $3.941 \mathrm{MeV}$ states, respectively), so the observed $\gamma$ ray transitions could be attributed to either candidate. However, since the $1680 \mathrm{keV}$ transition is not observed, the $10.707 \mathrm{MeV}$ state is more likely as a candidate for the $10.70 \mathrm{MeV}$ state populated by other $\left({ }^{6} \mathrm{Li}, d\right)$ experiments. Note that the unknown peak around $1550 \mathrm{keV}$ in the $\gamma$ spectrum does not correspond to the transition.

Taking the observed $\gamma$-rays into consideration, it is likely that multiplets near both $10.36 \mathrm{MeV}$ and $10.7 \mathrm{MeV}$ are present. However, the identifying $\gamma$-ray transitions 
are not seen, e.g., the $1003 \mathrm{keV}$ transition, in coincidence with the $E_{x}=10.7-11.1 \mathrm{MeV}$ region (where half of the strength of a state around $10.7 \mathrm{MeV}$ is expected to lie). This indicates that population of states near $10.7 \mathrm{MeV}$ is weak. This is supported by both Giesen et al. and Talwar et al., who report the $10.36 \mathrm{MeV}$ as being the strongest of the three observed states.

Further assignment to specific states is not possible given the energy resolution and ambiguous origin of the observed $\gamma$ rays. Similarly, we made no attempt to extract angular distributions due to the complicated and uncertain mix of multiple states in this region.

\section{1. $E_{x}=10.7-11.1 \mathrm{MeV}$}

States observed in the past $\left({ }^{6} \mathrm{Li}, d\right)$ experiments are the $10.805\left(1^{-}\right)$(or 10.823) and $10.949\left(1^{-}\right) \mathrm{MeV}$. The $\gamma$-ray spectrum shows only a single strong transition at $1808 \mathrm{keV}$. This indicates that there is a direct transition from the initial state to the $1808 \mathrm{keV}$ state. This agrees with both the 10.805 and $10.949 \mathrm{MeV}$ states observed by Longland et al. [33] (78\% and 95\% via the 1808 $\mathrm{keV}$, respectively). It also rules out the possibility of the 10.823 MeV state being strongly populated since comparable 1129 and $1808 \mathrm{keV}$ transitions are expected from that state, according to the findings of Lotay et al. [45], who observed that the $10.823 \mathrm{MeV}$ state $\left(2^{+}\right)$decays via the $5.476 \mathrm{MeV}$ state, which should generate 1157, 1808, and $2510 \mathrm{keV} \gamma$ rays (assuming the $10.823 \rightarrow 5.746 \mathrm{MeV}$ transition is dominant). In contrast, only the $1808 \mathrm{keV}$ line is present in our spectrum.

It was not possible to draw any further conclusions about the relative intensities of the 10.805 and 10.953 $\mathrm{MeV}$ states composing the peak. Similarly, we did not attempt to construct an angular distribution due to the uncertain mix of states in this region.

$$
\text { 12. } E_{x}=11.1-11.5 \mathrm{MeV}
$$

This region includes excitation energies above the neutron decay threshold, and here (in the lower-middle panel of Fig. 10) we show only $\gamma$-ray transitions in coincidence with ${ }^{26} \mathrm{Mg}$ recoils. The astrophysically-important peak at $E_{x}=11.32 \mathrm{MeV}$ in the deuteron spectrum can be clearly observed, consistent with Giesen et al. and Talwar et al. According to past measurements [34,67], this state decays directly via the $1808 \mathrm{keV}$ state $(11.318 \rightarrow 1.808)$ with $47 \pm 4 \%$, through the $11.318 \rightarrow 7.060 \rightarrow$ GS scheme $(19 \pm 1 \%)$, or through the $11.318 \rightarrow 7.060 \rightarrow 3588$ scheme $(18 \pm 1 \%)$, or $11.318 \rightarrow 7.060 \rightarrow 2938$ scheme $(5.3 \pm 0.4 \%)$, or $11.318 \rightarrow 7.060 \rightarrow 1808$ scheme $(11 \pm 0.8 \%)$. All together, the expected $1808 \mathrm{keV} \gamma$-ray probability from this state is $81 \pm 5 \%$. Considering the $\gamma$-ray branchings, the $\sim 170$ deuteron events in this peak in coincidence with ${ }^{26} \mathrm{Mg}$ recoils, and the $5 \% \gamma$-ray detection efficiency at $1808 \mathrm{keV}$, we expect to see $7 \pm 11808 \mathrm{keV}$ counts from the 11.32
$\mathrm{MeV}$ state. This is consistent with the $10 \pm 3$ counts seen for this peak. The next-most-probable transition from the $11.32 \mathrm{MeV}$ state is the $1129 \mathrm{keV}$ line $(2938 \rightarrow 1808)$. This has an intensity $<10 \%$ of the total $1808 \mathrm{keV}$ intensity and hence should not be observable above background, which is consistent with our spectrum $(\leq 3$ counts after background subtraction).

Concerning other candidate peaks in this region, Lotay et al. observed that the $E_{x}=11.17 \mathrm{MeV}$ level decays via the $5.476 \mathrm{MeV}$ state, which eventually leads to 1129 (69\%), 1157 (51\%), 2510 (51\%), and $1808 \mathrm{keV} \mathrm{(} \mathrm{100 \% )}$ $\gamma$ rays [45]. Since we do not observe measurable 1129, $1157 \mathrm{keV}$ and $2510 \mathrm{keV}$ transitions $(2-3,2-3$ and 0 counts at most, respectively), it appears that this state is weakly populated compared to the $11.32 \mathrm{MeV}$. This conclusion is consistent with our earlier publication [49], which set an upper limit of 0.15 on the $\alpha$ spectroscopic factor from an analysis of the ${ }^{25} \mathrm{Mg}$-gated deuteron spectrum (without considering $\gamma$ rays). However, it should be noted that the branching ratio of the transition $(11.17 \mathrm{MeV} \rightarrow 5.476$ $\mathrm{MeV}$ ) is unknown. Thus, assuming the possibility that the decay mode is not dominant, it is not possible to draw a firm conclusion. Lotay et al. also reported that the $E_{x}=11.08 \mathrm{MeV}$ state decays via the $4.318 \mathrm{MeV}$ state, which eventually generates 2510 and $1808 \mathrm{keV} \gamma$ rays. We do not observe a transition at $2510 \mathrm{keV}$ (either in the $E_{x}=10.7-11.1 \mathrm{MeV}$ region.) Although branching ratios of the respective decay transitions are not available, assuming the decay via the $4.318 \mathrm{MeV}$ state is dominant, this state appears to also be only weakly populated compared to the $E_{x}=11.32 \mathrm{MeV}$. This is consistent with both Giesen et al. and Talwar et al., who also failed to observe this state $[40,42]$. However, the state was strongly populated and observed in the sub-Coulomb $\left({ }^{6} \mathrm{Li}, d\right)$ and $\left({ }^{7} \mathrm{Li}\right.$, t) measurements of Jayatissa et al [46].

$$
\text { 13. } E_{x}=11.6-12.1 \mathrm{MeV}
$$

Peaks in this region are exclusively neutron unbound and hence the corresponding $\gamma$-ray spectrum (the last two panels of Fig. 10) is gated on ${ }^{25} \mathrm{Mg}-d$ coincidences. Because the first excitate state of ${ }^{25} \mathrm{Mg}$ is $585 \mathrm{keV}, \gamma$ transitions should not appear below $E_{x}=11.68 \mathrm{MeV}\left(S_{n}+0.585\right.$ $\mathrm{MeV})$. In particular, we note that the strongly-populated peak at $E_{x}=11.83 \mathrm{MeV}$ comes in coincidence with a 585 $\mathrm{keV} \gamma$-ray transition by $\left({ }^{6} \mathrm{Li}, d n_{1} \gamma\right)$. We also note that the $585 \mathrm{keV}$ state is an isomer with a 3.38 ns half life [67]. Therefore, the average position of ${ }^{25} \mathrm{Mg}$ ions when the $\gamma$-rays are emitted is $\sim 10 \mathrm{~cm}$ off from the target position along the beam direction $(z)$. Given the dimension of our HPGe clover detectors $(z= \pm 5 \mathrm{~cm})$, a large portion of the $\gamma$-rays are emitted outside the detector array. As a result, the efficiency for observing the delayed $\gamma$ rays is significantly lower than for prompt decays. Nevertheless, $\sim 5$ counts were observed for the transition, which is significantly more than the expected $\sim 0.7$ counts based on observed $\sim 900$ counts of deuterons, the reported $n_{1} / n_{0}$ 
ratio of $\sim 0.01$ [34], and $8 \% \gamma$-ray efficiency for prompt $\gamma$-rays (note we have not attempted to calculate the reduction in efficiency resulting from the 3.38 ns half life). This suggests that either the $n_{1} / n_{0}$ ratio for this state is significantly larger than reported in Ref. [34], or that there are multiple states besides the $11.83 \mathrm{MeV}$ state populated in this region. It also should be noted that two transitions (389 and $974 \mathrm{keV}$ ) from the second excited state (974) keV appear in the figure as well. The $\gamma$ decay channel opens above $E_{x}=12.06 \mathrm{MeV}$. Thus, these two $\gamma$ transitions come from the higher $E_{x}(>12.1 \mathrm{MeV})$ states. On the other hand, the excessively counted 585 $\mathrm{keV}$ transition comes mostly from the $E_{x}=11.8-11.9$ $\mathrm{MeV}$ region (central part of the $E_{x}=11.6-12.1 \mathrm{MeV}$ region).

The $E_{x}=11.83 \mathrm{MeV}$ state is reported to have $J^{\pi}=1^{-}$ or $2^{+}$, or $3^{-}$by a direct $(\alpha, n)$ measurement [34], while [42] included the possibility of $0^{+}$. Therefore, we compared the angular distribution with these DWBA curves. $J^{\pi}=0^{+}$or $1^{-}$is likely based on the angular distribution. If it is the same resonance $\left(E_{\alpha}=1434 \mathrm{keV}\right)$ observed by [34], the state has most likely $1^{-}$(see Section IV B for more details).

\section{4. $E_{x}>12.1 \mathrm{MeV}$}

A large peak was observed at $E_{x} \sim 13.15 \mathrm{MeV}$. A number of ${ }^{25} \mathrm{Mg}$ around the energy range are outside the MDM entrance window due to evaporations of high energy neutrons. No information regarding the resonances and neutron decays $\left(\left(\alpha, n_{0}\right),\left(\alpha, n_{1}\right),\left(\alpha, n_{2}\right)\right.$, etc. $)$ are available from the past ${ }^{22} \mathrm{Ne}(\alpha, n)$ and $\left({ }^{6} \mathrm{Li}, d\right)$ experiments. Furthermore, $\Gamma_{\alpha}$ can become large enough to compete with neutron decays (see e.g., [33]), which needs coincidence measurements with ${ }^{22} \mathrm{Ne}$ recoils. Thus, both of the MDM's detection efficiency and the spectroscopic factor are difficult to reliably estimate. Moreover, given that the resonances are highly unbound $(\sim 2.5 \mathrm{MeV}$ above the $\alpha$ threshold) where the DWBA calculations are relatively unreliable, we did not analyze the deuteron angular distributions of any states from the energy region. The $\gamma$ spectrum from the energy range showed a large transition via the $1611 \mathrm{keV}$ state (the third exited state of ${ }^{25} \mathrm{Mg}$ ) as well as minor transitions via other low-lying states (1.964, 0.974, and $0.585 \mathrm{MeV})$, indicating a number of decays occur via $\left(\alpha, n_{3}\right)$.

\section{B. Relative $\alpha$ strength}

As mentioned, the $\alpha$ partial decay width, $\Gamma_{\alpha}$, of $\alpha$ unbound resonances within the Gamow window is the most important parameter determining the total ${ }^{22} \mathrm{Ne}+\alpha$ stellar reaction rate. For a given spin and resonance energy, this parameter is proportional to the $\alpha$ spectroscopic factor, $S_{\alpha}$. The spectroscopic factor is also an indicator of possible $\alpha$ cluster structures in both bound and unbound states in ${ }^{26} \mathrm{Mg}$. For a given spin, the spectroscopic factor can be extracted from the angular distributions shown in Fig. 9. Table V shows the extracted spectroscopic factors of the states which were clearly observed in the present experiment. Between excitation energies of 5.7-9.3 MeV, this is the first time that individual states have been identified and assigned spectroscopic factors.

As in Ref. [49], the $S_{\alpha}$ presented in Table V are normalized to the spectroscopic factor for the state at $E_{x}=11.318 \mathrm{MeV}$. The $S_{\alpha}$ for this state was calculated from the ratio $\Gamma_{\alpha} / \Gamma_{\alpha}^{(\text {s.p.) }}$, where the single-particle $\alpha$ width $\left(\Gamma_{\alpha}^{\text {(s.p. })}\right)$ was calculated numerically from the wave function used in the DWBA calculation. The $\alpha$ partial width was taken from the results of direct $(\alpha, \gamma)$ measurements, $\Gamma_{\alpha}=\omega \gamma_{(\alpha, \gamma)}\left(1+\Gamma_{n} / \Gamma_{\gamma}\right) /(2 J+1)$, with $\omega \gamma_{(\alpha, \gamma)}=$ $37 \pm 4 \mu \mathrm{eV}$ (the weighted average of Refs. [34, 47, 48]) and $\Gamma_{n} / \Gamma_{\gamma}=1.14 \pm 0.26$ from Ref. [49]. Separate normalizations assuming $J_{11318}^{\pi}=0^{+}$and $J_{11318}^{\pi}=1^{-}$were calculated and are presented in Table V. A separate normalization was also performed to the excited state at $4.332 \mathrm{MeV}$, which was reported as having $S_{\alpha} / S_{\alpha}^{(\text {g.s. })}=$ 0.29 in Ref. [84]. For this normalization, $S_{\alpha}^{(4332)}$ was fixed at 0.29 , effectively giving the same normalization as Ref. [84], which fixed $S_{\alpha}^{\text {(g.s.) }}=1.0$.

The spectroscopic factors and associated $\alpha$ partial widths (or upper limits) for states from 11.11-11.32 MeV have already been discussed in Ref. [49]. We note the presence of a number of possible strong $\alpha$ cluster states $\left(S_{\alpha} \sim 0.1-0.5\right)$ in this region. More detailed conclusions about these states would require additional studies that focus more directly on this region. For excitation energies below $5.7 \mathrm{MeV}$, we can make direct comparisons to Ref. [84] when normalizing to $S_{\alpha}^{\text {(g.s.) }} \equiv 1$. In this region, the spectroscopic factor for the $E_{x}=5.475 \mathrm{MeV}$ state agrees with Ref. [84], while spectroscopic factors for the $E_{x}=4.835$ and $4.901 \mathrm{MeV}$ states are 2-4 times larger than Ref. [84]. This latter difference likely results from the present treatment of the observed peak as a single state. In contrast, Ref. [84] treated their peak as a triplet and obtained separate $S_{\alpha}$ for individual states using a least-squares fit to their angular distribution. We note that the ratios of differential cross sections to the 4.3 and $4.9 \mathrm{MeV}$ states at $\theta_{C M} \sim 10^{\circ}$, are consistent for the two experiments $(\sim 1)$. As a result, we can claim reasonable agreement between observed spectra for states in this region, although the interpretations differ.

Above $E_{x}=11.32 \mathrm{MeV}$, we focus on the $\alpha$ partial width extracted for the strongly-populated resonance at $E_{x}=11.83 \mathrm{MeV}$. This resonance is the dominant contributor to the ${ }^{22} \mathrm{Ne}(\alpha, n)^{25} \mathrm{Mg}$ rate in the high-temperatures ( $T \sim 1 \mathrm{GK}$ ) realized during the $\mathrm{C}$-shell burning phase of the s-process. This state has been observed in a number of past direct $(\alpha, n)$ measurements, which report values of 606(90) $\mathrm{meV}$ [34], 1067(42) $\mathrm{meV}$ [36], and $1105(120) \mathrm{meV}$ [87]. These results are in poor statistical agreement $\left(\chi^{2} / \mathrm{NDF}=11\right)$. Hence, we calculate an inflated weighted average of the three measurements, 
930(170) meV, as prescribed by Longland et al. [33] and use this value for comparisons with the present data.

In Table VI, we show the resonance strength of this state extracted from the present data, calculated as $\omega \gamma \simeq(2 J+1) \Gamma_{\alpha}$. The calculations were done assuming both $0^{+}$and $1^{-}$spin-parity for the $E_{x}=11.32 \mathrm{MeV}$ state (used for normalization) and for separate $J^{\pi}$ values of $J^{\pi}=\left(1^{-}, 2^{+}, 3^{-}\right)$as reported in Ref. [34]. Taken at face value, the resonance strengths support a $2^{+}$assignment to the $11.83 \mathrm{MeV}$ state when compared with the direct-measurement strength of 930(170) meV. We stress, however, that these calculations assume only the 11.83 $\mathrm{MeV}$ state exists in the observed $\left({ }^{6} \mathrm{Li}, d\right)$ peak, whereas both the width of the observed peak and the intensity of coincident $585 \mathrm{keV} \gamma$ rays (Section IV A 13) indicate other states being populated in this energy region-for example at $E_{x}=11.89$ or $11.91 \mathrm{MeV}(\omega \gamma=410(40)$ and $1400(100) \mathrm{meV}$, respectively [33]). A similar conclusion was also made about the spectrum observed in Ref. [40]. As a result, the resonance strengths reported in Table VI are most conservatively treated as upper limits. Taken as such, comparison with the direct measurements rules out the $3^{-}$assignment and leaves a possibility for $1^{-}$or $2^{+}$. The $1^{-}$possibility is also consistent with the presentlyobserved angular distribution (see Fig. 9).

\section{ASTROPHYSICAL IMPLICATIONS}

To investigate the impact of our recent measurements on $s$-process nucleosynthesis, we have performed abundance calculations using the ${ }^{22} \mathrm{Ne}(\alpha, n){ }^{25} \mathrm{Mg}$ and ${ }^{22} \mathrm{Ne}(\alpha, \gamma){ }^{26} \mathrm{Mg}$ reaction rates presented in Ref. [49]. The calculations were performed using the post-processing nucleosynthesis code MPPNP [50], which was developed by the NuGrid collaboration. The code takes as an input the stellar evolution trajectories calculated by the one-dimensional stellar evolution code MESA [88]. The MESA trajectories employed in the present work represent a variety of initial stellar conditions and were prepared by the NuGrid collaboration in previous works $[22,89,90]$. In the present calculations, we only varied the ${ }^{22} \mathrm{Ne}(\alpha, n)^{25} \mathrm{Mg}$ and ${ }^{22} \mathrm{Ne}(\alpha, \gamma)^{26} \mathrm{Mg}$ reaction rates and kept the values of all other input parameters fixed. As a result, the present calculations only probe the impact of the ${ }^{22} \mathrm{Ne}+\alpha$ reactions on the final $s$-process abundances.

For the AGB stars with initial mass $\mathrm{M}=3$ and $5 M_{\odot}$, we consider abundances at the stellar surface after the last Third Dredge Up episode. For the massive star models, we focus on the pre-supernova abundances in the middle of the convective $\mathrm{C}$ shell once the $s$-process nucleosynthesis has finished and before the final core-collapsed supernova (CCSN) explosion. However, we also consider abundances at the end of the He core burning, to better understand the total nucleosynthesis.

In the first part of our calculations (Section VA), we show the sensitivity of the predicted $s$-process to variance in the ${ }^{22} \mathrm{Ne}+\alpha$ reaction rates as given in prior studies: Massimi et al [39] (where only an upper limit was given), Longland et al [33], Talwar et al [42], and Adsley et al. [91], as well as our previous publication [49]. These rates are hereafter referred to as MA17, LO12, TA16, AD21, and OT20, respectively. These calculations are made for 3,5 , and $25 M_{\odot}$ stars with metallicity $Z=0.02$ from the beginning of H-burning until the end of hydrostatic stellar evolution. The $3 M_{\odot}$ model is from Ref. [90], and the 5 and $25 M_{\odot}$ models are from Ref. [89]. Note that while $J^{\pi}=0^{+}$was adopted for the $E_{x}=11.32 \mathrm{MeV}$ resonance in our rates (see [49] for details), only marginal differences were observed from the results presented below when we used $J^{\pi}=1^{-}$for the resonance instead.

In the second part of the calculations (Section VB), we investigate the impact of hypothetical changes of selected ${ }^{22} \mathrm{Ne}+\alpha$ resonances on predicted final $s$-process abundances. In particular, we show the sensitivity of the $s$-process abundances to uncertainties in the $\alpha$ strength of three resonances $\left(E_{x}=11.112,11.171\right.$, and $\left.11.319 \mathrm{MeV}\right)$. These resonances were chosen for study because they have the potential to dominate one or both of the ${ }^{22} \mathrm{Ne}+\alpha$ rates in certain temperature regimes and furthermore, because the present literature is either lacking key information or contains discrepancies between studies.

\section{A. Impact of new reaction rates}

Figure 11 shows the isotopic abundances produced using different ${ }^{22} \mathrm{Ne}+\alpha$ reaction rates by LO12, TA16 and OT20 for 3 and $5 M_{\odot}$ with $\mathrm{Z}=0.02$, respectively. For all three cases, the "recommended" rates published in the respective papers were used in the calculations. Figure 12 shows the same abundances as Figure 11 but for $25 M_{\odot}$ at the end of He core burning and in the middle of $\mathrm{C}$ shell burning, respectively. Abundances are shown as overproduction factors, which are defined by $\log _{10}\left(X_{f i n} / X_{i n i}\right)$, where $X_{f i n}$ and $X_{i n i}$ represent the final and initial mass fractions, respectively. The mass fraction, $X$, is given by $A \times N$ where $A$ is the mass number of an isotope and $N$ is its abundance. The sum of all mass fractions from hydrogen up to bismuth is equal to unity. The initial abundance is solar-scaled as in Ref. [22], based on Ref. [92] and with the isotopic ratios from Ref. [93].

The largest impact from using different rates is generated in mass $A=60-90(Z=27-40)$ in all the models considered. The large overproduction factors of heavier elements in the $3 M_{\odot}$ model is predominantly driven by neutrons from ${ }^{13} \mathrm{C}(\alpha, n){ }^{16} \mathrm{O}$, not from the ${ }^{22} \mathrm{Ne}(\alpha, n){ }^{25} \mathrm{Mg}$ reaction. Thus, only marginal differences are generated by using different reaction rates in the mass region $(A>90)$. In this case, the contribution to heavy elements from ${ }^{22} \mathrm{Ne}(\alpha, n){ }^{25} \mathrm{Mg}$ reaction is generally more limited to isotopes near $s$-process branching points, that are affected by its short but high neutron flux. For a comprehensive list of branching points we refer to Ref. [26].

As a general trend, the LO12 rates produce the high- 
TABLE V. Excitation energies, spin-parities, and spectroscopic factors for ${ }^{26} \mathrm{Mg}$ states populated in the present $\left({ }^{6} \mathrm{Li}, d\right)$ experiment. Note the spectroscopic factors are obtained by assuming one single state dominates in a given multiplet peak. Three separate normalizations are used for the spectroscopic factors as explained in the text. When available, spectroscopic factors from Refs. [40, 42, 84] are also listed.

\begin{tabular}{|c|c|c|c|c|c|}
\hline$E_{x}(\mathrm{keV})$ & $J^{\pi}$ & $S_{\alpha}\left(J_{11318}=0\right)$ & $S_{\alpha}\left(J_{11318}=1\right)$ & $S_{\alpha} / S_{\alpha}^{,(\text {g.s. })}$ & Refs. $[40,42,84]$ \\
\hline $4332^{a b}$ & $2^{+}$ & $0.07(1)$ & $0.12(2)$ & $0.29(6)^{\mathrm{j}}$ & $0.29(4)^{\mathrm{k}}$ \\
\hline $4835,4901^{\mathrm{ab}}$ & $2^{+}, 4^{+}$ & $0.06(1), 0.11(2)$ & $0.11(2), 0.20(3)$ & $0.26(6), 0.48(10)$ & $0.06(2)^{\mathrm{k}}, 0.20(4)^{\mathrm{k}}$ \\
\hline $5476^{\mathrm{ab}}$ & $4^{+}$ & $0.03(1)$ & $0.05(1)$ & $0.12(4)$ & $0.08^{1}$ \\
\hline $5715^{\mathrm{ab}}$ & $4^{+}$ & $0.02(1)$ & $0.04(2)$ & $0.09(4)$ & \\
\hline $6745,6876^{\mathrm{ab}}$ & $2^{+}, 3^{-}$ & $0.12(2), 0.10(1)$ & $0.22(3), 0.17(3)$ & $0.52(11), 0.41(9)$ & \\
\hline $7348,7371^{\mathrm{abc}}$ & $3^{-}, 2^{+}$ & $0.30(3), 0.27(2)$ & $0.50(5), 0.47(4)$ & $1.27(21), 1.13(18)$ & \\
\hline $8036^{\mathrm{bcd}}$ & $2^{+}$ & $0.11(2)$ & $0.20(3)$ & $0.48(10)$ & \\
\hline $8201^{\mathrm{abc}}$ & $6^{+}$ & $0.17(2)$ & $0.29(4)$ & $0.71(14)$ & \\
\hline $8625^{\mathrm{abc}}$ & $5^{-}$ & $0.09(1)$ & $0.16(2)$ & $0.38(7)$ & \\
\hline $8937^{\mathrm{bcd}}$ & $2^{+}$ & $0.08(1)$ & $0.14(2)$ & $0.35(7)$ & \\
\hline $9325,9371^{\text {abce }}$ & $2^{+}, 4^{+}$ & $0.22(2), 0.21(2)$ & $0.38(3), 0.38(3)$ & $0.92(15), 0.91(15)$ & \\
\hline $9856^{\mathrm{a}}$ & $2^{+}$ & $0.06(1)$ & $0.10(2)$ & $0.25(5)$ & \\
\hline $11318^{\mathrm{cfgh}}$ & $0^{+}, 1^{-}$ & $0.31(5)$ & $0.18(3)$ & $1.32(28), 0.43(9)$ & $0.43^{\mathrm{l}}, 0.04^{\mathrm{m}}$ \\
\hline $11831^{\mathrm{fi}}$ & $1^{-}, 2^{+}$ & $0.42(6), 0.29(6)$ & $0.74(10), 0.51(10)$ & $1.79(35), 1.24(30)$ & $0.20^{\mathrm{n}}, 0.11^{\mathrm{n}}$ \\
\hline
\end{tabular}

a Excitation energies are adopted from [67]

b Observed by [84]. $E_{x}>5.715 \mathrm{MeV}$ are observed in this work by digitizing the spectrum from [84].

c Observed by [42]

d Excitation energies are adopted from [42]

e Observed by [85]

f Observed by [40]

g Observed by [46]

h Excitation energies and spin-parities are from [49]

${ }^{\mathrm{i}}$ Excitation energies are adopted from [40]

j normalized to [84], where $S_{\alpha}^{\text {(g.s.) }}=1$.

k from [84], normalized to $S_{\alpha}^{\text {(g.s.) }}=1$.

${ }^{1}$ from [42] assuming $J^{\pi}=1^{-}$.

$\mathrm{m}$ from [40] assuming $J^{\pi}=1^{-}$.

${ }^{\mathrm{n}}$ from [40]

TABLE VI. ${ }^{22} \mathrm{Ne}(\alpha, n){ }^{25} \mathrm{Mg}$ resonance strengths for the $E_{x}=11.83 \mathrm{MeV}$ state in ${ }^{26} \mathrm{Mg}$, deduced from the present data. Resonance strengths are presented in units of meV. The subscripts to the $\omega \gamma$ symbol denote the assumed $J^{\pi}$ assignment for the $11.83 \mathrm{MeV}$ state. Calculations for both $0^{+}$and $1^{-}$ spin-parities of the $E_{x}=11.32 \mathrm{MeV}$ state are included as indicated. For comparison, the inflated weighted average of direct-measurement resonance strengths $\omega \gamma=930(170) \mathrm{meV}$.

\begin{tabular}{cccc}
\hline \hline$J_{1132}^{\pi}$ & $\omega \gamma_{1-}$ & $\omega \gamma_{2+}$ & $\omega \gamma_{3-}$ \\
\hline $0^{+}$ & $4110(577)$ & $1175(235)$ & $259(52)$ \\
$1^{-}$ & $7230(1020)$ & $2075(414)$ & $377(75)$ \\
\hline \hline
\end{tabular}

est $s$-process abundances, while our rates show the lowest $s$-process efficiency. Using the TA16 rates, we obtain $s$ process abundances that are somewhere in between. The main difference of the TA16 rate from LO12 is the treatment of the $E_{x}=11.17 \mathrm{MeV}$ resonance. While LO12 did not include the resonance, TA16 assigned a large $(\alpha, \gamma)$ strength in addition to the $(\alpha, n)$ upper limit imposed by Ref. [36]. Thus, TA16 rates, especially the $(\alpha, \gamma)$ rate, are much larger than LO12 at the $s$-process temperature range. In the $3 M_{\odot}$ star, the differences between
LO12 and TA16 are minor; however, the impact becomes clearly visible in the 5 and $25 M_{\odot}$ stars. This is because the TA16's large $(\alpha, \gamma)$ rate is more efficient in depleting ${ }^{22} \mathrm{Ne}$ in competition with the $(\alpha, n)$ reaction, leading to the reduced neutron flux. The main difference of our rates from LO12 is the reduced $(\alpha, n)$ strength in the $E_{x}=11.32 \mathrm{MeV}$ resonance, by about a factor of 3 . This generates the lower efficiency in producing neutrons for the $s$-process shown in Figure 11 and 12 by using the ${ }^{22} \mathrm{Ne}+\alpha$ rates presented in this work. In the $25 M_{\odot}$ star, it should be noted that the overproduction factors by TA16 nearly remain unchanged in the $\mathrm{C}$ shell burning compared to the He core burning, while the overproduction factors by LO12 and OT20 are largely enhanced. This is because ${ }^{22} \mathrm{Ne}$ are drastically consumed during the He core burning using TA16, thus $s$-process in the $\mathrm{C}$ shell burning is largely suppressed.

Figure 13 shows the isotopic abundances of representative s-only nuclei (see e.g., [94]), produced by using the same three rates considered for Figure 11 and 12. Additionally, we have considered the upper-limit ${ }^{22} \mathrm{Ne}+\alpha$ rates given in MA17. Calculations using the AD21 rates were also performed with results indistinguishable from the OT20 rates. This is expected since both studies use 
very similar ${ }^{22} \mathrm{Ne}+\alpha$ rates, with only minor rate differences resulting from the treatment of low energy resonances. As a result, the AD21 calculations are not shown in Figure 13. For all the rates excluding MA17, error bars are provided for the $s$-process abundances. Uncertainties are estimated by using the combination of the upper limit of the $(\alpha, n)$ and of the lower limit of the $(\alpha, \gamma)$ (yielding the highest $s$-process efficiency), and the lower limit of the $(\alpha, n)$ with the upper limit of the $(\alpha, \gamma)$ (yielding the lowest $s$-process efficiency).

Overall, the upper limit by MA17 is consistent with all the other rates. Indeed, the MA17 rate includes contributions from all possible low energy resonances $\left(E_{x}=11.1-\right.$ 11.25 MeV), all of which are overwhelmed by the $(\alpha, n)$ strength and the $(\alpha, \gamma)$ is negligible. In particular, the lowest resonance at $E_{x}=11.11 \mathrm{MeV}$ is the main source of the enhanced ${ }^{22} \mathrm{Ne}(\alpha, n){ }^{25} \mathrm{Mg}$ rate.

Compared to the rates given in TA16, our recommended rates produce up to a factor of 3 lower $s$-process abundances. However, the two sets of $s$-process calculations are consistent within the rate uncertainties given in the two studies. On the other hand, we obtain a reduction up to a factor of 10 compared to the results using the LO12 rates. In this case, the variation between the two sets of abundances is not compatible with the errors given. In general, we may conclude that within the uncertainties of our new ${ }^{22} \mathrm{Ne}+\alpha$ rates, we obtain a significant reduction in the $s$-process contribution to the galactic chemical evolution of elements between iron and the $s$-process peak of Sr, Y and Zr. The significance of these effects will be studied in a forthcoming paper.

\section{B. Sensitivity to $\alpha$ cluster strength}

In order to provide a complementary approach to assess the impact of the ${ }^{22} \mathrm{Ne}+\alpha$ rates provided in the previous section, we also calculated the abundances when varying strengths of key resonances at $E_{x}=11.11,11.17$, and $11.32 \mathrm{MeV}$. Starting with the reaction rates presented in this work, we made calculations assuming four different conditions: 1 ) change $\Gamma_{n} / \Gamma_{\gamma}$ of the $11.32 \mathrm{MeV}$ resonance from 1.14 to $3.78 ; 2$ ) insert a strong $\alpha$ cluster state at $11.17 \mathrm{MeV}$ with significant $\gamma$-ray decay probability; 3) increase $\Gamma_{\alpha}$ of the $11.11 \mathrm{MeV}$ resonance to 13.5 $\mathrm{neV}$; and 4) assume the $11.11 \mathrm{MeV}$ resonance does not exist at all. Each of these changes is intended to probe the impact of existing discrepancies from the literature.

Specifically, condition 1 probes the impact of the discrepancies between the present work (together with Refs. $[46,49])$ and direct $(\alpha, n)$ measurements. The former established an $(\alpha, n)$ strength of $42(11) \mu \mathrm{eV}$, while the latter have a weighted average of $140(30) \mu \mathrm{eV}$ for the same. In both cases, the $(\alpha, \gamma)$ strength is taken to be 37(4) $\mu \mathrm{eV}$.

Condition 2 probes the impact of a strong $\alpha$ cluster state at $11.17 \mathrm{MeV}$, as claimed by TA16 but absent in the present work and others $[40,46,49]$. In this condi- tion, we inserted an "observed" (not upper limit) $(\alpha, \gamma)$ resonance at $11.17 \mathrm{MeV}$ with $\omega \gamma_{(\alpha \gamma)}=660 \pm 90 \mathrm{neV}-$ the largest possible strength reported in TA16. Note that we modified the energy of this resonance slightly compared to TA16, placing it at $11.171 \mathrm{MeV}$ instead of $11.167 \mathrm{MeV}$. We did this to account for the findings of MA17, which suggest a state with large $\gamma$-decay probability at $E_{x}=11.171 \mathrm{MeV}$. For the $(\alpha, n)$ reaction, we increased the upper-limit strength of this resonance to $\omega \gamma<60 \mathrm{neV}$, as reported by Jaeger et al. [36].

Together, conditions 3 and 4 probe the impact of uncertainties on the $\alpha$ width, and hence $\omega \gamma_{(\alpha, n)}$ of the $2^{+}$ resonance at $11.11 \mathrm{MeV}$. Condition 3 probes the impact of a stronger $11.11 \mathrm{MeV}$ resonance, up to the $\omega \gamma<13.5$ $\mathrm{neV}\left(\Gamma_{\alpha}<2.7 \mathrm{neV}\right)$ limit used to determine the upperlimit rates reported in MA17 [95] (note that MA17's limits on the resonance strength were extracted from Ref. [36]). To evaluate the maximum possible impact of this resonance, we treated it as an "observed" resonance in the Monte Carlo calculations, with a strength of $\omega \gamma=13.5 \mathrm{neV}$ (as opposed to an "unobserved" resonance with the strength sampled from a Porter-Thomas distribution with an upper limit of $13.5 \mathrm{neV}$ ). As such, this calculation evaluates the impact of there being a strong $11.11 \mathrm{MeV}$ resonance, with a strength just below the observation threshold of Ref. [36].

In contrast to condition 3 , condition 4 probes the impact of there being a negligibly small $\alpha$ width for the $11.11 \mathrm{MeV}$ resonance, to the point where it can be removed from the rate calculations entirely.

Figure 14 and 15 show the resulting isotopic abundances when the reaction rates defined by conditions 1-3 are used. The abundances from each of the three conditions are plotted as a ratio to the baseline rate from OT20 (Texas A\&M (TAMU) abundances). Figure 14 shows abundances for 3 and $5 M_{\odot}$ stars, and Figure 15 shows $25 M_{\odot}$ abundances following both He-core and Cshell burning as indicated. Figure 16 shows the same abundance ratios as the previous two figures but for $s$ only nuclei exclusively. This figure also includes the calculations using the condition 4 rate. These are nearly indistinguishable from the OT20 results and thus were excluded from the previous two plots.

For the $3 M_{\odot}$ model, the abundances are mainly sensitive to the changes in condition 3 . The sensitivity to condition 1 is minor (25\% increase on average) and the impact of condition 2 is negligible. The insensitivity to condition 2 mirrors the indistinguishable results between the LO12 and TA16 rates in the previous section (Figures 11 and 13). Evidently, the abundances for the 3 $M_{\odot}$ model are insensitive to a strong $(\alpha, \gamma)$ resonance at $\sim 11.17 \mathrm{MeV}$. The minor sensitivity to the condition 1 rates is limited to $A<90$ nuclides, which again is consistent with the abundance differences from the previous section when comparing our rates and those of LO12 and TA16, which used the higher $11.32 \mathrm{MeV}$ strength established in direct measurements. The large sensitivity to condition 3 -increasing the $11.11 \mathrm{MeV}$ resonance 

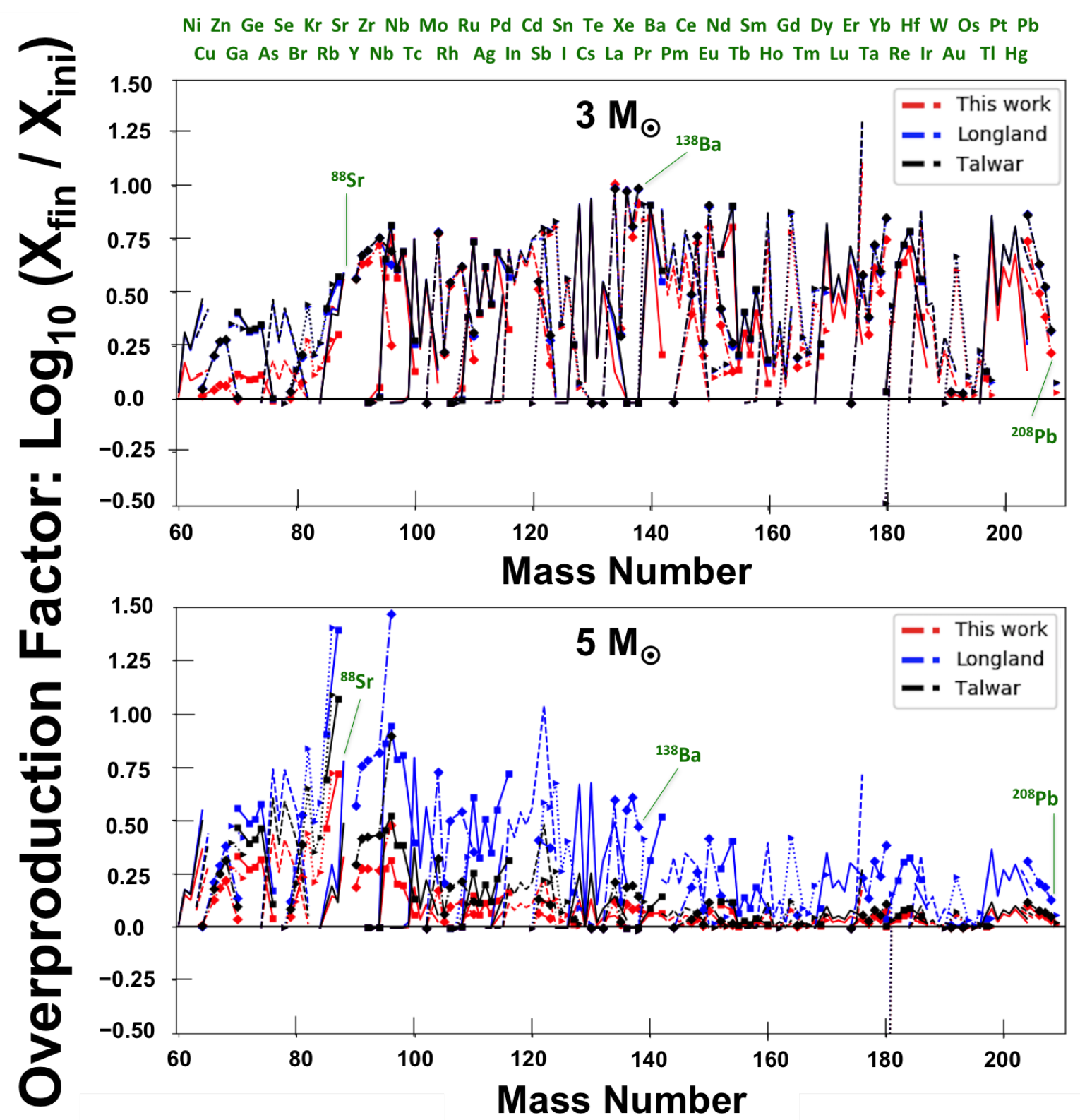

FIG. 11. Calculated $s$-process overproduction factors for the 3 and $5 M_{\odot}$ stars using various available ${ }^{22} \mathrm{Ne}(\alpha, \mathrm{n})$ and ${ }^{22} \mathrm{Ne}(\alpha, \gamma)$ rates. See text for details. Isotopes of the same elements are connected by adjoining lines (some key $s$-process peak isotopes $\left({ }^{88} \mathrm{Sr},{ }^{138} \mathrm{Ba}\right.$, and $\left.{ }^{208} \mathrm{~Pb}\right)$ are labeled for clarification).

strength to MA17's upper limit-mirrors the previous section's differences between the present rates and those of MA17. The complete insensitivity to the changes of condition 4-removing the $11.11 \mathrm{MeV}$ resonancedemonstrates that the strength of this resonance is already well constrained in our rates when considering its impact on $3 M_{\odot}$ stars.

For the $5 M_{\odot}$ model, conditions 1 and 3 both result in a large increase in predicted abundances. Both rate changes have as much as a factor 3-4 influence on the abundances for certain elements, e.g. Mo. For this model, condition 2 decreases the predicted abundances by a fac- tor of two at most. This reduction is expected because the increased $(\alpha, \gamma)$ rate, resulting from the strong 11.17 $\mathrm{MeV}$ resonance, competes with $(\alpha, n)$ for fuel. Hence, the stronger $(\alpha, \gamma)$ rate reduces neutron production via ${ }^{22} \mathrm{Ne}(\alpha, n)$. The increase in the $11.11 \mathrm{MeV}$ resonance strength (condition 3) again increases abundances substantially. The removal of the $11.11 \mathrm{MeV}$ resonance entirely (condition 4) again results in negligible abundance changes.

For the $25 M_{\odot}$ model, the largest sensitivity is observed for condition 3, similar to the other models, although now the impact is as large as a factor $\sim 100$ for 
Ni Zn Ge Se Kr Sr Zr Nb Mo Ru Pd Cd Sn Te Xe Ba Ce Nd Sm Gd Dy Er Yb Hf W Os Pt Pb

$\mathrm{Cu}$ Ga As Br Rb Y Nb Tc Rh Ag In Sb I Cs La Pr Pm Eu Tb Ho Tm Lu Ta Re Ir Au Tl Hg
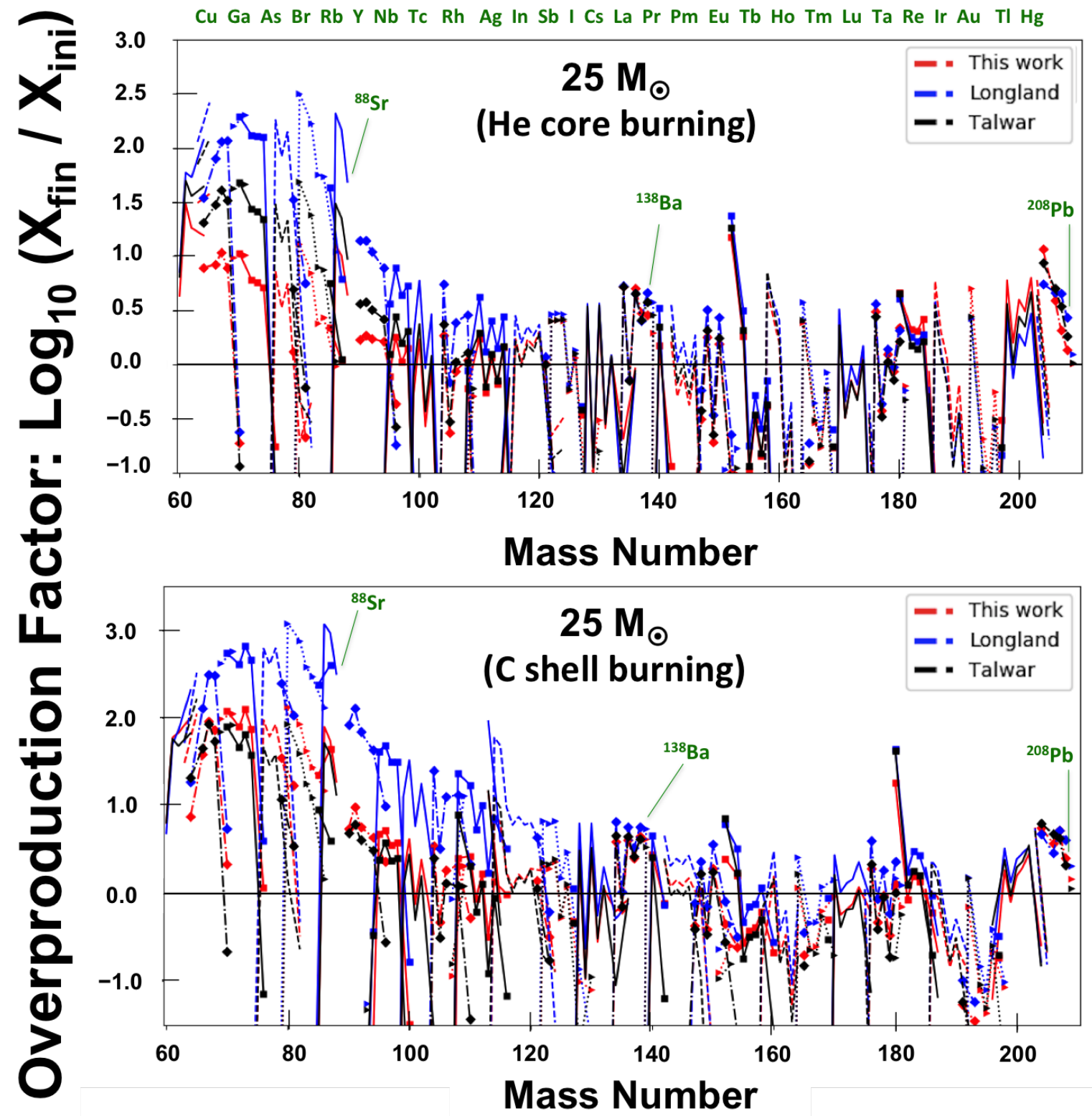

FIG. 12. Calculated $s$-process overproduction factors for the $25 M_{\odot}$ star using various available ${ }^{22} \mathrm{Ne}(\alpha, \mathrm{n})$ and ${ }^{22} \mathrm{Ne}(\alpha, \gamma)$ rates. See text for details. Isotopes of the same elements are connected by adjoining lines (some key $s$-process peak isotopes $\left({ }^{88} \mathrm{Sr}\right.$, ${ }^{138} \mathrm{Ba}$, and ${ }^{208} \mathrm{~Pb}$ ) are labeled for clarification).

elements near $A=100$. Condition 1 leads to up to a factor $\sim 10$ increase in production for the lighter nuclides. The impact of condition 2 is significant, leading to a large (factor $\lesssim 50$ ) decrease in abundances for elements near $A=80$. Condition 4 again has a minor impact, although a perceptible decrease (factor $\sim 1.25$ ) in abundances is now present for the lightest nuclides.

\section{SUMMARY}

Natural parity states in ${ }^{26} \mathrm{Mg}$ were studied from $E_{x}=4$ $14 \mathrm{MeV}$ using the ${ }^{22} \mathrm{Ne}\left({ }^{6} \mathrm{Li}, d\right)$ reaction in inverse kinematics. Coincidence tagging with the ${ }^{25,26} \mathrm{Mg}$ recoils identified the first-order decay channels (neutron or $\gamma$ ray) of the populated states. The resulting spectra were interpreted with the help of coincident $\gamma$-ray measurements and the results of past ${ }^{22} \mathrm{Ne}\left({ }^{6} \mathrm{Li}, d\right)$ measurements. This information was used to identify the most probable states contributing to the deuteron-reconstructed 

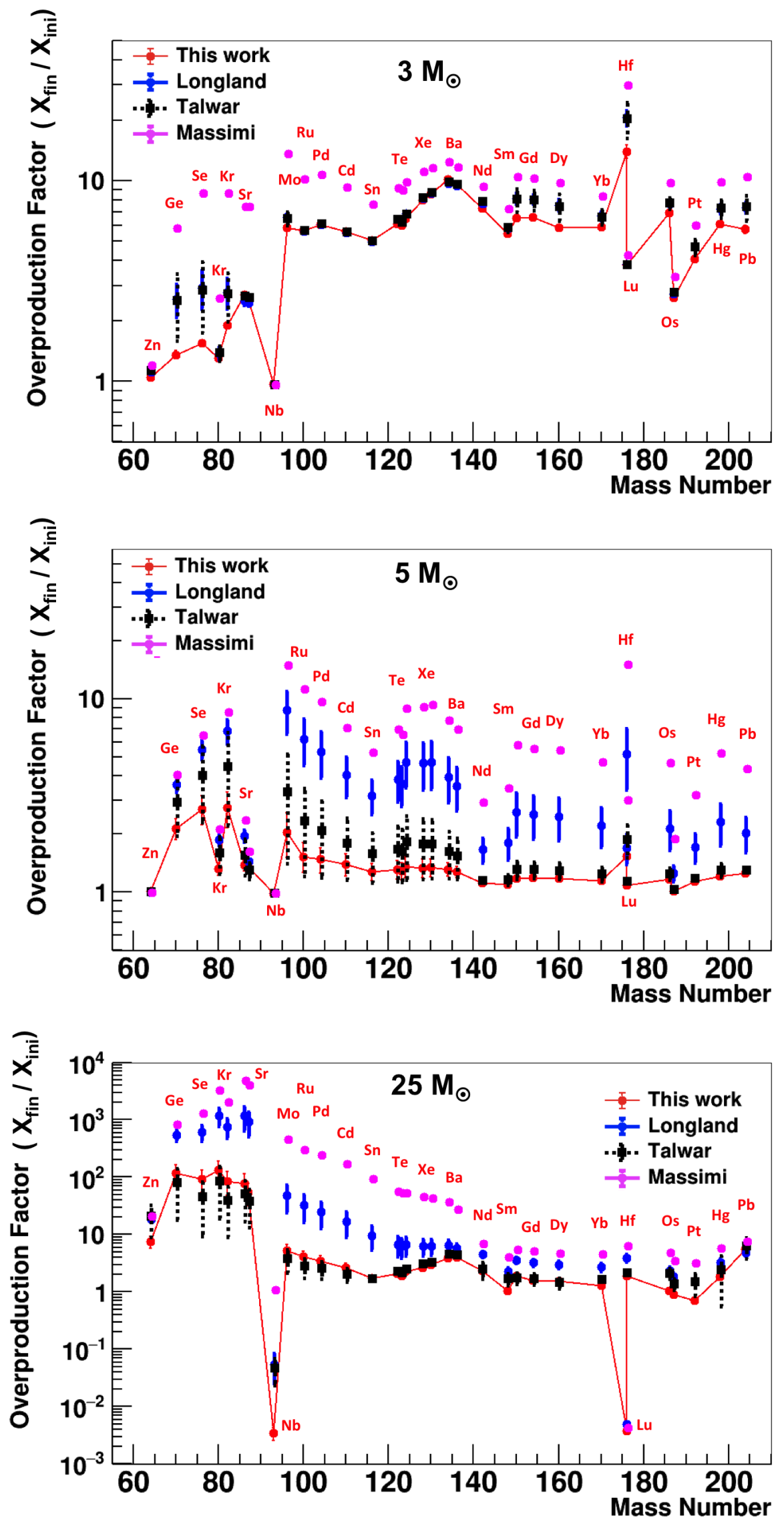

FIG. 13. Calculated $s$-process overproduction factors using various available ${ }^{22} \mathrm{Ne}(\alpha, \mathrm{n})$ and ${ }^{22} \mathrm{Ne}(\alpha, \gamma)$ rates, for s-only nuclei. Top, middle, and bottom panels correspond to 3, 5, and 25 (in the middle of the $\mathrm{C}$ shell burning) $M_{\odot}$ cases, respectively. Note that the rate given by Massimi et al. [39] is their upper limit (see texts for details). 
Ni Zn Ge Se Kr Sr Zr Nb Mo Ru Pd Cd Sn Te Xe Ba Ce Nd Sm Gd Dy Er Yb Hf W Os Pt Pb

$\mathrm{Cu}$ Ga As Br Rb Y Nb Tc Rh Ag In Sb I Cs La Pr Pm Eu Tb Ho Tm Lu Ta Re Ir Au TI Hg
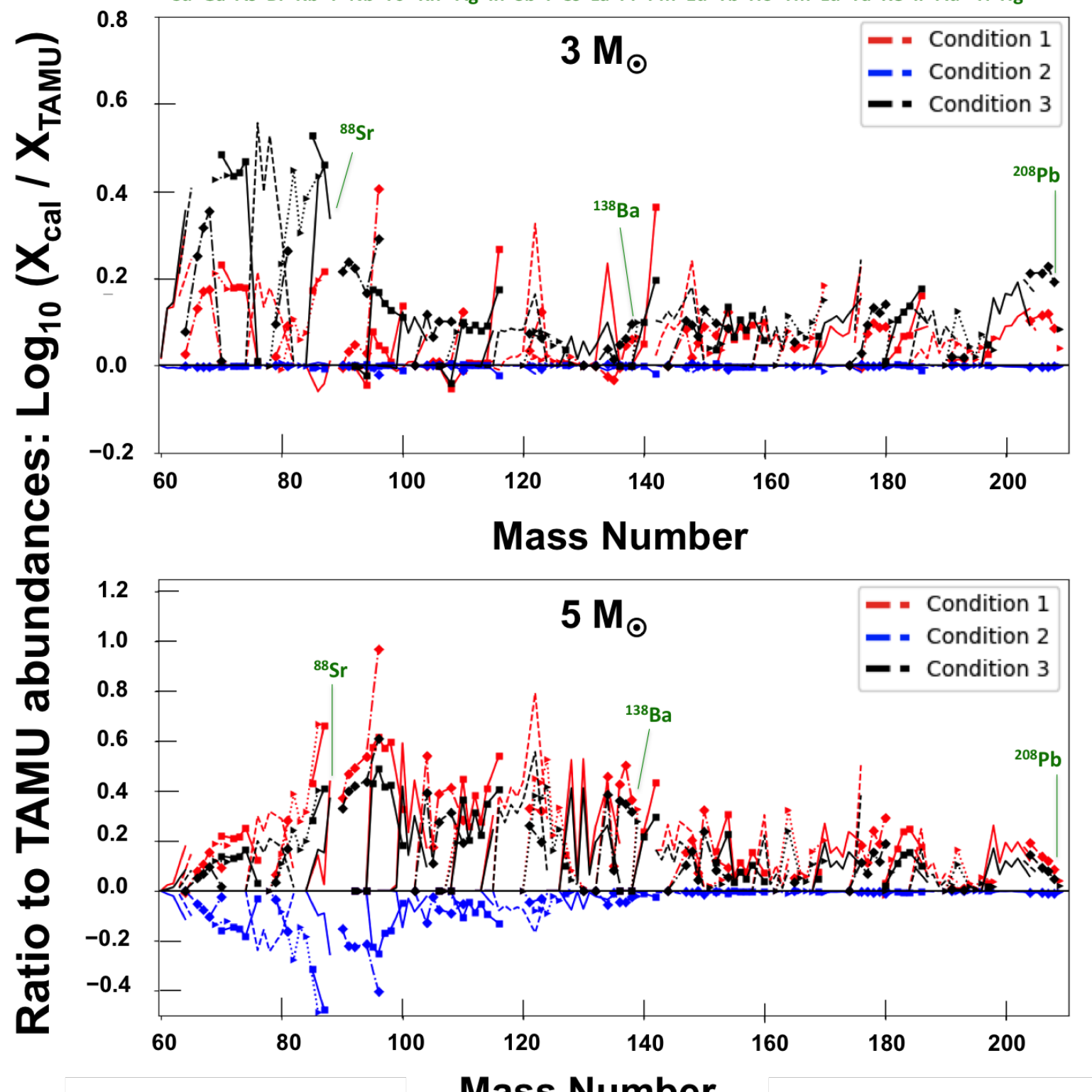

FIG. 14. Ratios of calculated $s$-process abundances to TAMU abundances for the 3 and $5 M_{\odot}$ stars (see Figure 11) using ${ }^{22} \mathrm{Ne}(\alpha, \mathrm{n})$ and ${ }^{22} \mathrm{Ne}(\alpha, \gamma)$ rates in which strength of some resonances are changed. See text for details. Isotopes of the same elements are connected by adjoining lines (some key $s$-process peak isotopes $\left({ }^{88} \mathrm{Sr},{ }^{138} \mathrm{Ba}\right.$, and $\left.{ }^{208} \mathrm{~Pb}\right)$ are labeled for clarification).

excitation-energy spectrum, which did not have sufficient resolution to separate all states populated in the reaction. Spins and relative spectroscopic factors were assigned to the identified states through comparison of angular distributions with DWBA calculations. The ${ }^{22} \mathrm{Ne}\left({ }^{6} \mathrm{Li}, t\right)$ reaction was also analyzed. States likely to be strongly populated in this reaction were identified, and $\left({ }^{6} \mathrm{Li}, t\right)$ spectroscopic factors were reported for the first time.

The analysis of the $\left({ }^{6} \mathrm{Li}, d\right)$ data from this experiment, in the region of interest for the astrophysical $s$-process, led to the construction of new Monte-Carlo reaction rates for the stellar ${ }^{22} \mathrm{Ne}(\alpha, n)$ and ${ }^{22} \mathrm{Ne}(\alpha, \gamma)$ reactions [49]. These rates were used to calculate predicted $s$-process abundances for a range of elements, using three different stellar models with $M / M_{\odot}=3,5$, and 25 and $\mathrm{Z}=0.02$. These calculations show the impact of the ${ }^{22} \mathrm{Ne}+\alpha$ reaction rates on the $s$-process abundances, using constraints from presently-available nuclear physics information. Using the new rates established by the results of the present experiment, we observe a reduction in the overabundance 
Ni Zn Ge Se Kr Sr Zr Nb Mo Ru Pd Cd Sn Te Xe Ba Ce Nd Sm Gd Dy Er Yb Hf W Os Pt Pb $\mathrm{Cu}$ Ga As Br Rb Y Nb Tc Rh Ag In Sb I Cs La Pr Pm Eu Tb Ho Tm Lu Ta Re Ir Au Tl Hg
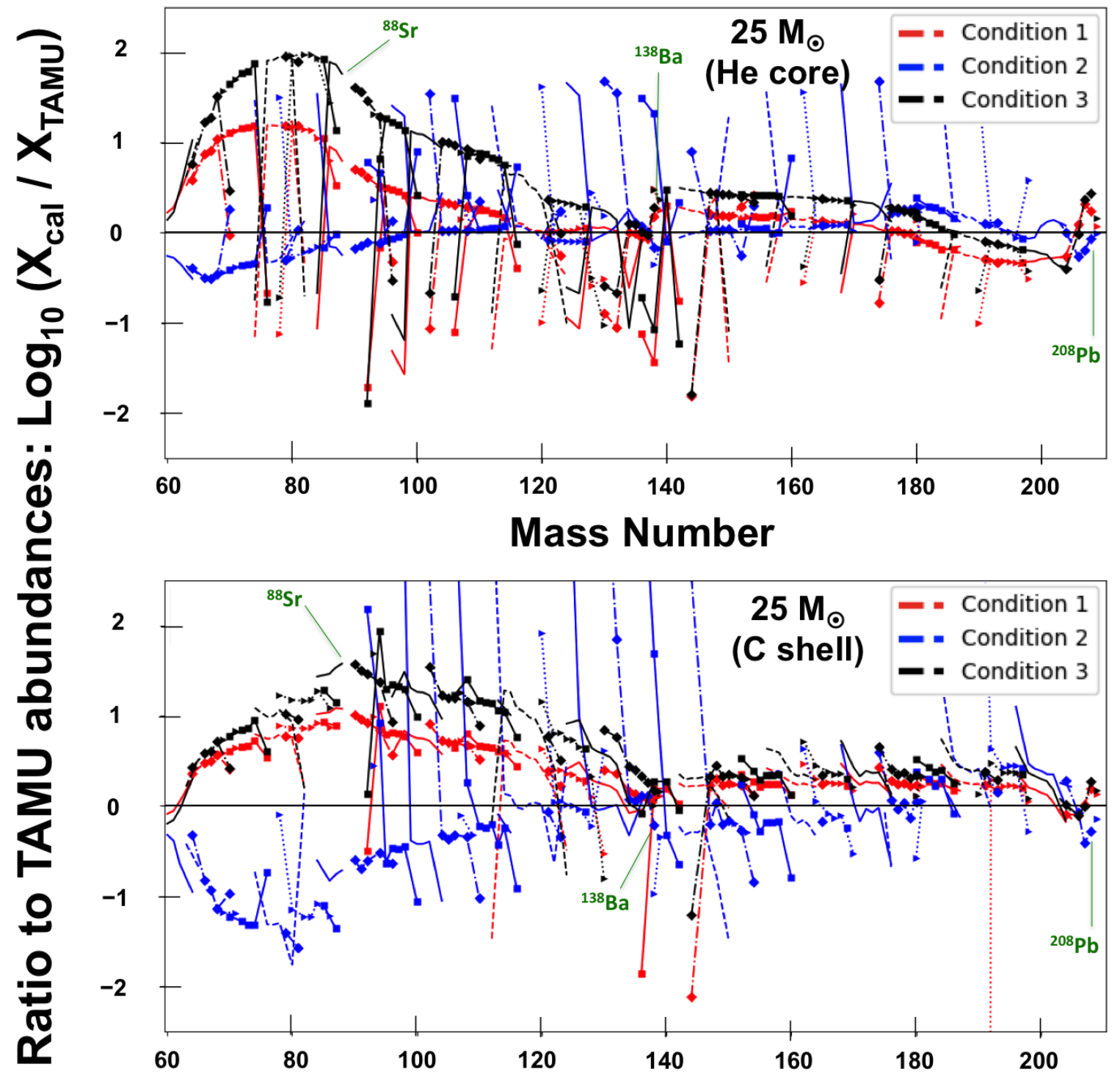

Mass Number

FIG. 15. Ratios of calculated s-process abundances to TAMU abundances for the $25 M_{\odot}$ (see Figure 12$)$ using ${ }^{22} \mathrm{Ne}(\alpha, \mathrm{n})$ and ${ }^{22} \mathrm{Ne}(\alpha, \gamma)$ rates in which strength of some resonances are changed. See text for details. Isotopes of the same elements are connected by adjoining lines (some key $s$-process peak isotopes $\left({ }^{88} \mathrm{Sr},{ }^{138} \mathrm{Ba}\right.$, and $\left.{ }^{208} \mathrm{~Pb}\right)$ are labeled for clarification).

of certain elements of up to a factor of 3 and 10 compared to the earlier rates published in Talwar et al. (TA16) [42] and Longland et al. (LO12) [33], respectively. These difference are mainly observed in mass range $A=60-90$, for all three stellar mass models.

We also used our stellar models to investigate the impact on $s$-process nucleosynthesis of outstanding uncertainties or literature discrepancies on selected, key ${ }^{22} \mathrm{Ne}+\alpha$ resonances. These calculations used the present Monte Carlo rates as a baseline and subsequently varied strengths of the resonances at $E_{x}=11.11,11.17$, and 11.32 MeV. The results highlight the strong astrophysical impact of the factor $\sim 3$ reduction in the $E_{x}=11.32$ $\mathrm{MeV}(\alpha, n)$ strength, which resulted from the present experiment. The calculations also established that the discrepancies between the present work (along with others $[33,40,46])$ and TA16 concerning the presence of a strong $(\alpha, \gamma)$ resonance at $11.17 \mathrm{MeV}$ are relatively minor except for the $25 M_{\odot}$ stars. Finally, the calculations established that the presently-determined upper limit on 

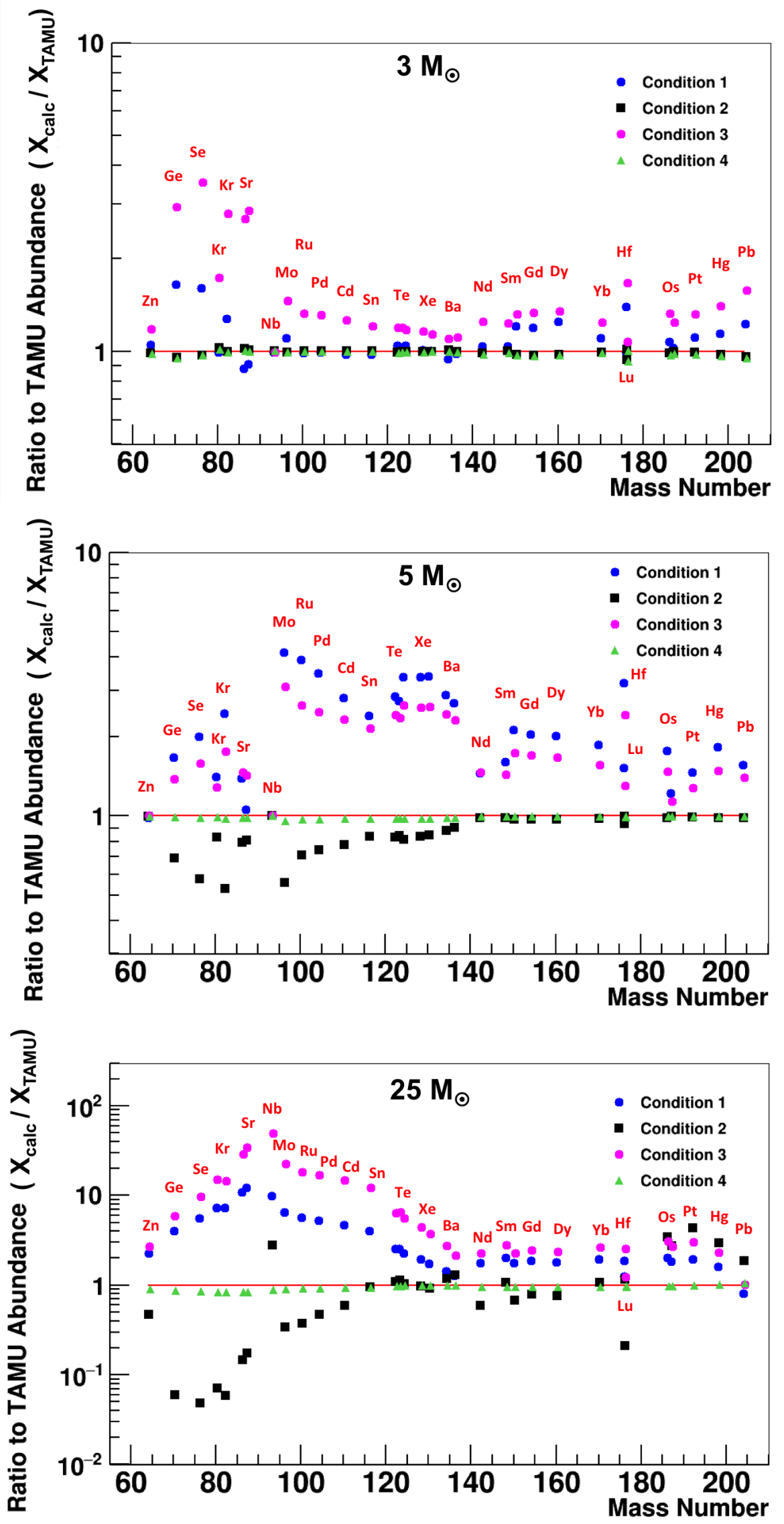

FIG. 16. Calculated $s$-process abundance ratio to TAMU abundances for s-only nuclei (see Figure 14 and 15$)$ using ${ }^{22} \mathrm{Ne}(\alpha, \mathrm{n})$ and ${ }^{22} \mathrm{Ne}(\alpha, \gamma)$ rates in which strength of some resonances are changed. Top, middle, and bottom panels correspond to 3 , 5 , and 25 (in the middle of the $\mathrm{C}$ shell burning) $M_{\odot}$ cases, respectively. 
the strength of the $2^{+}$resonance at $11.11 \mathrm{MeV}$ is sufficient to constrain $s$-process nucleosynthesis across all models. At the same time, all three models predict a strong enhancement in $s$-process abundances if a resonance is found just below the observation threshold of Ref. [36] around $E_{x}=11.11 \mathrm{MeV}$. This highlights the significant importance of this resonance to $s$-process nucleosynthesis. All together, the calculations point to the 11.11 and $11.32 \mathrm{MeV}$ resonances as being the most important for the $s$-process. As a result, we encourage future experiments targeted at independently corroborating the strengths of these resonances established by the present experiment.

\section{ACKNOWLEDGMENTS}

We express our thanks to the technical staff at the Texas A\&M University Cyclotron Institute. Financial support for this work was provided by the US De- partment of Energy, award Nos. DE-FG02-93ER40773 and DE-SC0018980, the US National Nuclear Security Administration, award No. DE-NA0003841. SO acknowledges support from the TAMU CIRD fund. JAT, WNC, and GL acknowledge support from the UK STFC, award no. ST/L005743/1. MP acknowledges support from NuGrid, JINA-CEE (NSF Grant PHY-1430152), STFC (through the University of Hulls Consolidated Grant ST/R000840/1), and ongoing access to viper, the University of Hull High Performance Computing Facility. MP also acknowledges the support from the "Lendület-2014" Programme of the Hungarian Academy of Sciences (Hungary). UB acknowledges support from the European Research Council ERC-2015-STG Nr. 677497. We also acknowledge the ChETEC COST Action (CA16117), supported by the European Cooperation in Science and Technology, the CheTEC-INFRA (European Unions Horizon 2020 research and innovation program, grant agreement No 101008324) and IReNA (USA National Science Foundation under Grant No. OISE1927130).
[1] F. Kappeler, R. Gallino, S. Bisterzo, and W. Aoki. Rev. Mod. Phys., 83(157), 2011.

[2] John J. Cowan, Christopher Sneden, James E. Lawler, Ani Aprahamian, Michael Wiescher, Karlheinz Langanke, Gabriel Martínez-Pinedo, and Friedrich-Karl Thielemann. Origin of the heaviest elements: The rapid neutron-capture process. Reviews of Modern Physics, 93(1):015002, January 2021.

[3] J. J. Cowan and W. K. Rose. Production of ${ }^{14} \mathrm{C}$ and neutrons in red giants. Astrophys. J., 212:149-158, February 1977.

[4] P. A. Denissenkov, F. Herwig, U. Battino, C. Ritter, M. Pignatari, S. Jones, and B. Paxton. APJ Lett., 2(834), 2017.

[5] L. F. Roberts, S. E. Woosley, and R. D. Hoffman. Integrated Nucleosynthesis in Neutrino-driven Winds. Astrophys. J., 722(1):954-967, October 2010.

[6] A. Arcones and F. Montes. Production of Light-element Primary Process Nuclei in Neutrino-driven Winds. Astrophys. J., 731(1):5, April 2011.

[7] M. Arnould and S. Goriely. Phys. Rep., 384(1):1-84, 2003.

[8] C. Travaglio, T. Rauscher, A. Heger, M. Pignatari, and C. West. APJ, 854(18), 2018.

[9] U. Battino, M. Pignatari, C. Travaglio, C. LedererWoods, P. Denissenkov, F. Herwig, F. Thielemann, and T. Rauscher. MNRAS, 497:4981-4998, 2020.

[10] LIGO Scientific Collaboration et al. APJ Lett., 848(L12), 2017.

[11] M. R. Drout, A. L. Prito, and B. J. et al. Shappee. Science, 358:1570-1574, 2017.

[12] M. Arnould, S. Goriely, and K. Takahashi. Phys. Rep., 450(97), 2007.

[13] C. Sneden, J. J. Cowan, and Gallino. Annu. Rev. Astron. Astrophys., 46(241), 2008.

[14] Z. Y. Bao, H. Beer, Kappeler, Voss F., F., and K. Wisshak. Atom. Dat. Nucl. Dat. Tab., 76:70-154, 2000.
[15] A. I. Karakas, D. A. Garcia-Hernandez, and M. Lugaro. APJ, 751(8), 2012.

[16] O. Straniero, R. Gallino, and S. Cristallo. Nucl. Phys. A, 777:311, 2006.

[17] Roberto Gallino, Claudio Arlandini, Maurizio Busso, Maria Lugaro, Claudia Travaglio, Oscar Straniero, Alessandro Chieffi, and Marco Limongi. Evolution and Nucleosynthesis in Low-Mass Asymptotic Giant Branch Stars. II. Neutron Capture and the S-Process. Astrophys. J., 497(1):388-403, April 1998.

[18] F. Käppeler, R. Gallino, S. Bisterzo, and Wako Aoki. The s process: Nuclear physics, stellar models, and observations. Reviews of Modern Physics, 83(1):157-194, January 2011.

[19] T. Rauscher, A. Heger, R. D. Hoffman, and S. E. Woosley. Nucleosynthesis in Massive Stars with Improved Nuclear and Stellar Physics. Astrophys. J., 576(1):323-348, September 2002.

[20] Lih-Sin The, Mounib F. El Eid, and Bradley S. Meyer. sProcess Nucleosynthesis in Advanced Burning Phases of Massive Stars. Astrophys. J., 655(2):1058-1078, February 2007.

[21] M. Pignatari, R. Gallino, M. Heil, M. Wiescher, F. Käppeler, F. Herwig, and S. Bisterzo. The Weak sProcess in Massive Stars and its Dependence on the Neutron Capture Cross Sections. Astrophys. J., 710(2):15571577, February 2010.

[22] M. Pignatari, F. Herwig, R. Hirschi, M. Bennett, G. Rockefeller, C. Fryer, F. X. Timmes, C. Ritter, A. Heger, S. Jones, U. Battino, A. Dotter, R. Trappitsch, S. Diehl, U. Frischknecht, A. Hungerford, G. Magkotsios, C. Travaglio, and P. Young. APJ, 225:2, 2016.

[23] James G. Peters. Nucleosynthesis by the s-PROCESS in Stars of 9 and 15 Solar Masses. Astrophys. J., 154:225, October 1968.

[24] I. Iben. APJ, 196(525), 1975.

[25] Amanda I. Karakas and John C. Lattanzio. The Dawes 
Review 2: Nucleosynthesis and Stellar Yields of Low- and Intermediate-Mass Single Stars. pasa, 31:e030, July 2014.

[26] S. Bisterzo, R. Gallino, F. Käppeler, M. Wiescher, G. Imbriani, O. Straniero, S. Cristallo, J. Görres, and R. J. deBoer. The branchings of the main s-process: their sensitivity to $\alpha$-induced reactions on ${ }^{13} \mathrm{C}$ and ${ }^{22} \mathrm{Ne}$ and to the uncertainties of the nuclear network. MNRAS, 449(1):506-527, May 2015.

[27] C. M. Raiteri, M. Busso, R. Gallino, and G. Picchio. SProcess Nucleosynthesis in Massive Stars and the Weak Component. II. Carbon Burning and Galactic Enrichment. Astrophys. J., 371:665, April 1991.

[28] F. Kaeppeler, M. Wiescher, U. Giesen, J. Goerres, I. Baraffe, M. El Eid, C. M. Raiteri, M. Busso, R. Gallino, M. Limongi, and A. Chieffi. Reaction Rates for $18 \mathrm{O}$ ( alpha , gamma ) $22 \mathrm{Ne}, 22 \mathrm{Ne}$ ( alpha, gamma ) $26 \mathrm{Mg}$, and $22 \mathrm{Ne}($ alpha , n) $25 \mathrm{Mg}$ in Stellar Helium Burning and s-Process Nucleosynthesis in Massive Stars. Astrophys. J., 437:396, December 1994.

[29] A. Chieffi, M. Limongi, and O. Straniero. APJ, 737(502), 1998.

[30] Samuel W. Jones, Heiko Möller, Chris L. Fryer, Christopher J. Fontes, Reto Trappitsch, Wesley P. Even, Aaron Couture, Matthew R. Mumpower, and Samar Safi-Harb. ${ }^{60} \mathrm{Fe}$ in core-collapse supernovae and prospects for X-ray and gamma-ray detection in supernova remnants. $M N$ $R A S$, 485(3):4287-4310, May 2019.

[31] W. Wang, M. J. Harris, R. Diel, H. Halloin, B. Cordier, A. W. Strong, K. Kretschmer, P. Knodlseder, Jean, G. Lichti, J. P. Roques, S. Schanne, A. von Kienlin, G. Weidenspointner, and C. Wunderer. Astron. \& Astrophys., 469(3):1005-1012, 2007.

[32] M. Lugaro, U. Ott, and Á. Kereszturi. Radioactive nuclei from cosmochronology to habitability. Progress in Particle and Nuclear Physics, 102:1-47, September 2018.

[33] R. Longland, C. Iliadis, and A. I. Karakas. Phys. Rev. C, 85(065809), 2012.

[34] K. Wolke, V. Harms, H.W. Becket, J.W. Hammer, K.L. Kratz, C. Rolfs, U. Schroder, H.P. Trautvetter, M. Wiescher, and A. Wohr. Z. Phys. A, 334:491-510, 1989.

[35] H.W. Drotleff, A. Denker, J.W. Hammer, H. Knee, S. Kuchler, D. Streit, C. Rolfs, and H.P. Trautvetter. Z. Phys. A, 338:367-368, 1991.

[36] M. Jaeger, R. Kunz, A. Mayer, J. W. Hammer, G. Staudt, K. L. Kratz, and Pfeiffer B. Phys. Rev. Lett, 87(20), 2001.

[37] P. Koehler. Phys. Rev. C, 66(055805), 2002.

[38] C. Massimi, P. Koehler, and S. et al. Bisterzo. Phys. Rev. C, 85(044615), 2012.

[39] C. Massimi, S. Altstadt, and J. et al. Andrzejewski. Phys. Let. B, 768:1-6, 2017.

[40] U. Giesen, C.P. Browne, J. Gorres, S. Graff, C. Iliadis, H.P. Trautvetter, M. Wiescher, W. Harms, K.L. Kratz, B. Pfeiffer, R.E. Azuma, M. Buckby, and J.D. King. Nucl. Phys. A, 561:95-111, 1993.

[41] R. Longland, C. Iliadis, G. Rusev, A. P. Tonchev, R. J. deBoer, J. Gorres, and Wiescher M. Phys. Rev. C, 80(055803), 2009.

[42] R. Talwar, T. Adachi, G. P. A. Berg, L. Bin, S. Bisterzo, M. Couder, R. J. deBoer, X. Fang, H. Fujita, Y. Fujita, J. Gorres, K. Hatanaka, T. Itoh, T. Kadoya, A. Long, K. Miki, D. Patel, M. Pignatari, Y. Shimbara, A. Tamii, M. Wiescher, T. Yamamoto, and M. Yosoi. Phys. Rev.
C, 93(055803), 2016.

[43] P. Adsley, J. W. Brummer, K. C. W. Li, D. J. MarinLambarri, N. Y. Kheswa, L. M. Donaldson, R. Neveling, P. Papka, L. Pellegri, L. C. V. Pesudo, Pool, Smit F. D., and van Zyl J. J. Phys. Rev. C, 96(055802), 2017.

[44] P. Adsley, J.W. Brummer, T. Faestermann, S.P. Fox, F. Hammache, R. Hertenberger, A. Meyer, R. Noveling, D. Seiler, N. de Sereville, and H.-F. Wirth. Phys. Rev. C, 97(045807), 2018.

[45] G. Lotay, D. T. Doherty, D. Seweryniak, S. AlmarazCalderon, M. P. Carpenter, C. J. Chiara, H. M. David, C. R. Hoffman, R. V. F. Janssens, A. Kankainen, T. Lauritsen, R. Wilkinson, P. J. Woods, , and S. Zhu. Eur. Phys. J. A, 109(55), 2019.

[46] H. Jayatissa, G.V. Rogachev, V.Z. Goldberg, E. Koshchiy, G. Christian, J. Hooker, S. Ota, B.T. Roeder, A. Saastamoinen, O. Trippella, S. Upadhyayula, and E. Uberseder. Phys. Let. B, 802(135267), 2020.

[47] M. Jaeger. The reaction ${ }^{22} \mathrm{Ne}(\alpha, n)^{25} \mathrm{Mg}$, the key neutron source in massive stars. $\mathrm{PhD}$ thesis, Universität Stuttgart, 2001.

[48] S. Hunt, C. Iliadis, A. Champagne, L. Downen, and A. Cooper. Phys. Rev. C, 045804:99, 2019.

[49] S. Ota, G. Christian, G. Lotay, W.N. Catford, E.A. Bennett, D.T. Dede, S. Doherty, S. Hallam, J. Hooker, C. Hunt, H. Jayatissa, A. Matta, M. Moukaddam, G. V. Rogachev, A. Saastamoinen, J. A. Tostevin, S. Upadhyayula, and Wilkinson R. Phys. Lett. B, 802(135256), 2020.

[50] F. Herwig, R. Andrassy, N. Annau, O. Clarkson, B. Cote, A. D'Sa, S. Jones, B. Moa, J. O'Connell, D. Porter, C. Ritter, and P. Woodward. APJ Supple. Lett., 236:2, 2018.

[51] M. Labiche, W.N. Catford, R.C. Lemmon, C.N. Timis, R. Chapman, and N.A. etl. Orr. Nucl. Instr. Meth. A, 614:439-448, 2010.

[52] S. M. Brown. Neutron Shell Breaking in Neutron-Rich Neon Isotopes. PhD thesis, University of Surrey, Surrey, UK, 2010.

[53] D.M. Pringle, W.N. Catford, J.S. Winfield, D.G. Lewis, N.A. Jelley, K.W. Allen, and J.H. Coupland. Nucl. Instr. Meth. A, 245(2-3):230-247, 1986.

[54] A. Spiridon, E. Pollacco, B. T. Roeder, A. Saastamoinen, R. Chyzh, M. Dag, R. E. Tribble, L. Trache, G. Pascovici, and R. De Oliveira. Nucl. Instr. Meth. B, 376:364-368, 2016.

[55] A. Spiridon, E. Pollacco, A. Saastamoinen, M. Dag, B. Roeder, R. E. Tribble, L. Trache, G. Pascovici, B. Mehl, and R. de Oliveirad. Nucl. Instr. Meth. A, 943(162461), 2019.

[56] O. B. Tarasov and D. Bazin. http://lise.nscl.msu.edu/lise.html.

[57] S.R. Lesher, L. Phair, L.A. Bernstein, D.L. Bleuel, J.T. Burke, J.A. Church, P. Fallon, J. Gibelin, N.D. Scielzo, M. Wiedeking. Nucl. Instr. Meth. A, 621(1-3):286291, 2010.

[58] G. F. Knoll. Radiation Detection and Measurement. John Wiley and Sons, New York, 4th edition, 2010.

[59] H. F. Lutz, J. J. Wesolowski, Hansen L. F., and S. F. Eccles. Nucl. Phys. A, 95:591, 1967.

[60] A. J. Howard, J. O. Pronko, and C. A. Whitten. Nucl. Phys. A, 152:317, 1970.

[61] W.W. Daehnick, J.D. Childs, and Vrcelj Z. Phys. Rev. C, 21(6), 1980 . 
[62] H. An and C. Cai. Phys. Rev. C, 73(054605), 2006.

[63] I.J. Thompson. Comp. Phys. Rep., 7:167-212, 1988.

[64] J. A. Tostevin. University of Surrey version of the code TWOFNR (of M. Toyama, M. Igarashi and N. Kishida), http://nucleartheory.eps.surrey.ac.uk/NPG/code.htm, 1972.

[65] C. M. Perey and F. G. Perey. Atom. Dat. Nucl. Dat. Tab., 17:1-101, 1976.

[66] A. Matta, P. Morfouace, N. de Sereville, F. Flavigny, M. Labiche, and R. Shearman. J. Phys. G, 045113:43, 2016.

[67] National Nuclear Data Center website. http://www.nndc.bnl.gov.

[68] G. Bassani, T. H. Kruse, N. Saunier, and G. Souchere. Phys. Lett., 30B:9, 1969.

[69] G. Bassani, N. Saunier, and B. M. Traore. J. De. Physique, 11:133, 1971.

[70] M. L. Avila, L. T. Baby, J. Belarge, N. Keeley, K. W. Kemper, E. Koshchiy, A. N. Kuchera, G. V. Rogachev, K. Rusek, and D. Santiago-Gozalez. Phys. Rev. C, 97(014313), 2018.

[71] A. D. Panagiotou and H. E. Gove. Nucl. Phys. A, 196:144-155, 1972.

[72] J. D. Garrett, H. G. Bingham, H. T. Fortune, and R. Middleton. Phys. Rev. C, 5(3):682, 1972.

[73] L. M. Martz, S. J. Sanders, P. D. Parker, and C. B. Dover. Phys. Rev. C, 20(4):1340-1346, 1979.

[74] R. A. Lindgren, R. G. Markham, and H. W. Fulbright. Phys. Lett., 48B:3, 1974.

[75] C. W. Woods, N. Stein, and J. W. Sunier. Phys. Rev. C, 17:1, 1978.

[76] H. Ropke, G. Hammel, W. Brendler, P. Betz, and V. Glattes. Z. Phys. A, 266:55-64, 1974.

[77] F. Heidinger, P. Betz, W. Brendler, F. Glaz, A. Hoffmann, H. Ropke, and B.H. Wildenthal. Z. Phys. A, 338(23), 1991.

[78] I. Hamamoto and Ben R. Mottelson. Scholarpedia, 7 (4)(10693), 2012.

[79] F. Meurders and G. De Korte. Nucl. Phys. A, 249:205214, 1975.

[80] S. Hinds, R. Middleton, and G. Parry. Proc. Phys. Soc. LXXI, I, 1957.
[81] P. A. Butler, A. J. Brown, L. L. Green, A. N. James, C. J. Lister, J. D. MacArthur, P. J. Nolan, and J. F. Sharpey-Shafer. J. Phys. G, 1(6), 1975.

[82] J. E. Christiansson, J. Dubois, H. Roth, and L. Jarneborn. Physica Scripta., 14:193-196, 1976.

[83] X. Li, C. Liang, and C. Cai. Nucl. Phys. A, 789:103-113, 2007.

[84] N. Anantaraman, H.E. Dove, J. Toke, and J.P. Draayer. Nucl. Phys. A, 279:474-492, 1977.

[85] C. Ugalde, A. E. Champagne, S. Daigle, C. Iliadis, R. Longland, J. R. Newton, E. Osenbaugh-Stewart, J. A. Clark, C. Deibel, A. Parikh, P. Parker, and C. Wrede. Phys. Rev. C, 76:025802, 2007.

[86] F Glatz, S. Norbert, E. Bitterwolf, A. Burkard, F. Heidinger, Th Kern, R. Lehmann, H. Ropke, J. Siefert, C. Schneider, and B.H. Wildenthal. Z. Phys. A, 324:187204, 1986.

[87] H.W. Drotleff, A. Denker, H. Knee, M. Soine, G. Wolf, J.W. Hammer, U. Greifre, C. Rolfs, and H.P. Trautvetter. APJ, 414:735-739, 1993.

[88] B. Paxton, L. Bildsten, A. Dotter, F. Herwig, P. Lesaffre, and F. Timmes. APJ, 192:1, 2010.

[89] C. Ritter, F. Herwig, S. Jones, M. Pignatari, C. Fryer, and R. Hirschi. Monthly Notice of Royal Astron. Soc., 480:538-571, 2018.

[90] U. Battino, A. Tattersall, C. Lederer-Woods, F. Herwig, P. Denissenkov, R. Hirschi, R. Trappitsch, J. W. den Hartogh, and M. Pignatari. Monthly Notice of Royal Astron. Soc., 489:1082, 2019.

[91] P. Adsley, U. Battino, A. Best, A. Caciolli, A. Guglielmetti, G. Imbriani, H. Jayatissa, M. La Cognata, L. Lamia, E. Masha, C. Massimi, S. Palmerini, A. Tattersall, and R. Hirschi. Phys. Rev. C, 103(015805), 2021.

[92] N. Grevesse and A. Noels. Origin and evolution of the elements. Astronomisches Rechen-Institue, Heidelberg, (15), 1993.

[93] K. Lodders. APJ, 591(1220), 2003.

[94] N. Prantzos, C. Abia, S. Cristallo, M. Limongi, and A. Chieffi. MNRAS, 491:1832-1850, 2020.

[95] C. Massimi. private communication. March 2019. 